\title{
Crossover between short- and long-range proximity effects in superconductor/ferromagnet/superconductor junctions with Ni-based ferromagnets
}

\author{
O. M. Kapran, ${ }^{1}$ T. Golod, ${ }^{1}$ A. Iovan $\odot,{ }^{1,2}$ A. S. Sidorenko ${ }^{3,4}$ A. A. Golubov,${ }^{5,6}$ and V. M. Krasnov $\circledast^{1,6, *}$ \\ ${ }^{1}$ Department of Physics, Stockholm University, AlbaNova University Center, SE-10691 Stockholm, Sweden \\ ${ }^{2}$ Department of Applied Physics, Royal Institute of Technology, SE-10691 Stockholm, Sweden \\ ${ }^{3}$ Institute of Electronic Engineering and Nanotechnologies ASM, MD2028 Kishinev, Moldova \\ ${ }^{4}$ I.S. Turgenev Orel State University, 302026 Orel, Russia \\ ${ }^{5}$ Faculty of Science and Technology and MESA+ Institute of Nanotechnology, University of Twente, 7500 AE Enschede, The Netherlands \\ ${ }^{6}$ Moscow Institute of Physics and Technology, State University, 141700 Dolgoprudny, Russia
}

(Received 17 November 2020; revised 19 February 2021; accepted 1 March 2021; published 15 March 2021)

\begin{abstract}
We study superconductor/ferromagnet/superconductor junctions with $\mathrm{CuNi}$, PtNi, or Ni interlayers. Remarkably, we observe that supercurrents through Ni can be significantly larger than through diluted alloys. The phenomenon is attributed to the dirtiness of disordered alloys leading to a short coherence length despite a small exchange energy. To the contrary, pure $\mathrm{Ni}$ is clean resulting in a coherence length as long as in a normal metal. Analysis of temperature dependencies of critical currents reveals a crossover from short (dirty) to long (clean) range proximity effects in $\mathrm{Pt}_{1-x} \mathrm{Ni}_{x}$ with increasing $\mathrm{Ni}$ concentration. Our results point out that structural properties of a ferromagnet play a crucial role for the proximity effect and indicate that conventional strong-but-clean ferromagnets can be advantageously used in superconducting spintronic devices.
\end{abstract}

DOI: 10.1103/PhysRevB.103.094509

\section{INTRODUCTION}

A competition between superconductivity and ferromagnetism leads to an unconventional proximity effect, studied both theoretically [1-18] and experimentally [19-41]. In strong ferromagnets $(\mathrm{F}), \mathrm{Fe}, \mathrm{Co}$, and $\mathrm{Ni}$ exchange energies, $E_{\text {ex }} \sim 1000 \mathrm{~K}$, are much larger than the energy gap, $\Delta \sim 1-10 \mathrm{~K}$, in low- $T_{c}$ superconductors (S). Therefore, spinsinglet Cooper pairs are usually broken at a very short-range $\sim 1 \mathrm{~nm}$ in $\mathrm{F}$, as shown by many experimental works $[21,26-$ $28,31,34,39,40]$. There are, however, reports about a longrange proximity effect (LRPE) (tens to hundreds of $\mathrm{nm}$ ) $[19,25,30,33,35]$, which is often ascribed to the spin-triplet order parameter that should be immune to the ferromagnetic order.

Interpretation of LRPE remains controversial. First, there is a seeming irreproducibility of experimental results, cf. Refs. [19,30,35] and [21,40]. Second, the triplet order should appear only in the noncollinear magnetic state $[6,7,11]$, the origin of which is often unclear for structures containing only one F layer. Although several subtle effects, such as quantum fluctuations [3], active interfaces [5], domains [28,38], inhomogeneities $[10,13,17]$, and spin-orbit coupling $[16,17]$ were

\footnotetext{
*Vladimir.Krasnov@fysik.su.se
}

Published by the American Physical Society under the terms of the Creative Commons Attribution 4.0 International license. Further distribution of this work must maintain attribution to the author(s) and the published article's title, journal citation, and DOI. Funded by Bibsam. suggested, they are difficult to confirm or control in experiment. Finally, the proximity effect depends on the electronic mean-free path (m.f.p.) $l_{e}$ and, thus, on the internal structure. In particular, it has been predicted, that in clean $\mathrm{F}$ even a singlet supercurrent should exhibit LRPE [2,8,12,14,15,24]. Experimental analysis of SFFS spin valves has shown that the singlet current is dominant for diluted F [36] and remains considerable even for pure $\mathrm{Ni}$ [41]. Clarification of LRPE mechanisms and the ways of controlling supercurrents in $\mathrm{S} / \mathrm{F}$ heterostructures is important both for fundamental understanding of unconventional superconductivity [42] and for application in superconducting spintronics [18,32,34,39,41].

Here we study nanoscale SFS Josephson junctions (JJ's) containing either diluted $\mathrm{Ni}$ alloys $\mathrm{Cu}_{1-x} \mathrm{Ni}_{x}$ and $\mathrm{Pt}_{1-x} \mathrm{Ni}_{x}$, $\mathrm{Cu} / \mathrm{Ni}$ bilayer or pure $\mathrm{Ni}$. Counterintuitively, we observe that the supercurrent density $J_{c}$ through $\mathrm{Ni}$ can be much larger than through diluted alloys with the same thickness. Using in situ absolute Josephson fluxometry (AJF), we demonstrate that $\mathrm{Ni}$ interlayers in our junctions exhibit full saturation magnetization as in bulk $\mathrm{Ni}$, which precludes the presence of extended dead magnetic layers. The clue to understanding of our results is obtained from the analysis of evolution of temperature dependencies, $J_{c}(T)$, in $\mathrm{Nb} / \mathrm{Pt}_{1-x} \mathrm{Ni}_{x} / \mathrm{Nb}$ JJ's with increasing $\mathrm{Ni}$ concentration. It shows that in diluted $\mathrm{Ni}$ alloys, $x \simeq 0.5$, the proximity effect is short range, despite a small $E_{\text {ex }}$, due to an extremely short m.f.p. is such atomically disordered alloys. To the contrary, pure Ni remains clean, facilitating ballistic Cooper pair transport and LRPE similar in scale to that in the normal metal Pt. Our results demonstrate that the proximity effect in ferromagnets depends not only on composition and $E_{\text {ex }}$ but also essentially on the internal structure. This may help to resolve some of the controversies around LRPE. We 
TABLE I. Parameters of junctions with $\mathrm{Ni}, \mathrm{Cu} / \mathrm{Ni}$ and $\mathrm{CuNi}$ interlayers: $d_{F}$ is the thickness of F-interlayer; the size defines junction area $A=L_{x} \times L_{y} ; R_{n}$ is the normal resistance of the junction; $\rho_{n}=R_{n} A / d$ is junction resistivity, for junctions with $\mathrm{Cu} / \mathrm{Ni}$ bilayer $d=d_{\mathrm{Cu}}+d_{\mathrm{Ni}}=$ $20 \mathrm{~nm}$, for the rest $d=d_{F} ; T$ is the temperature; $I_{c}$ is the maximum critical current, $J_{c}=I_{c} / A$ is the critical current density; $I_{c} R_{n}$ is the characteristic voltage. For some junctions values of $I_{c}, J_{c}$ and $I_{c} R_{n}$ at different $T$ are provided.

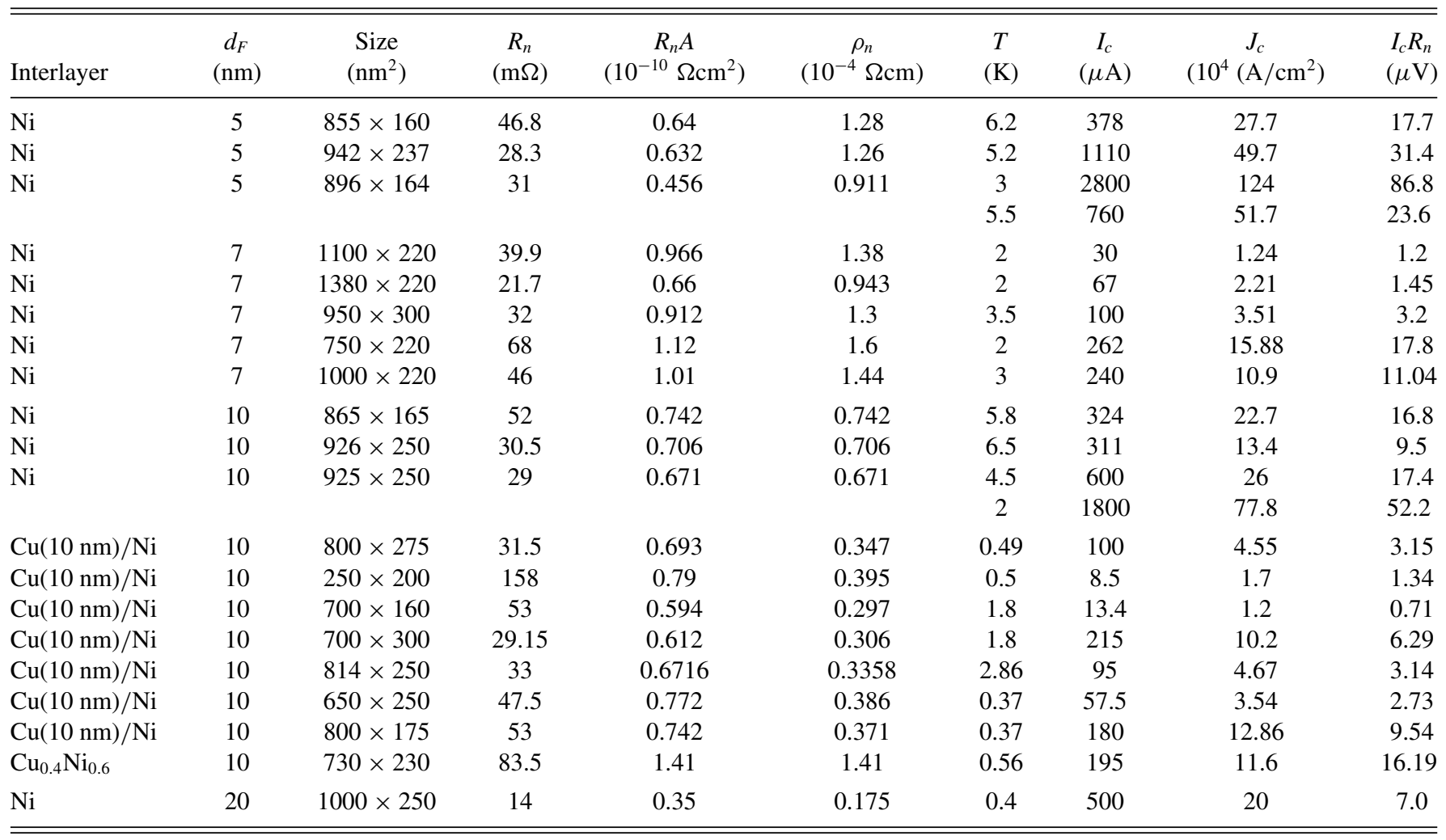

conclude that strong-but-clean ferromagnets may have advantages compared to weak-but-dirty for device applications.

The paper is organized as follows. In Sec. II we describe sample fabrication and experimental procedures. In Sec. III we discuss main experimental results, including Sec. III A, in situ magnetic characterization of $\mathrm{Ni}$ interlayers via AJF, and Sec. III B, analysis of temperature dependencies of critical currents, which reveals a crossover between clean (ballistic) and dirty (diffusive) transport. In the appendices we provide additional information about: Appendix A-film structure, Appendix B-junction characteristics, Appendix $\mathrm{C}$ - properties of $\mathrm{Nb} / \mathrm{Pt}_{1-x} \mathrm{Ni}_{x} / \mathrm{Nb}$ junctions, Appendix Dinterface resistances in $\mathrm{Nb} / \mathrm{Pt}_{1-x} \mathrm{Ni}_{x} / \mathrm{Nb}$ junctions, and Appendix $\mathrm{E}$ - extraction of magnetization curves from AJF analysis.

\section{SAMPLES AND EXPERIMENTAL}

We present data for nanoscale SFS junctions with $\mathrm{F}$ interlayers made of $\mathrm{Cu}_{0.4} \mathrm{Ni}_{0.6}$ and $\mathrm{Pt}_{1-x} \mathrm{Ni}_{x}$ alloys with $x=$ $0-1$, pure $\mathrm{Ni}$ and a $\mathrm{Cu} / \mathrm{Ni}(\mathrm{N} / \mathrm{F}, \mathrm{N}$-normal metal) bilayer. SFS multilayers were deposited by dc-magnetron sputtering in a single cycle without breaking vacuum. $\mathrm{Cu}_{1-x} \mathrm{Ni}_{x}$ films were deposited by cosputtering from $\mathrm{Cu}$ and $\mathrm{Ni}$ targets and the concentration was controlled by the corresponding sputtering rates. $\mathrm{Pt}_{1-x} \mathrm{Ni}_{x}$ films were deposited from composite targets with different areas of $\mathrm{Ni}$ and $\mathrm{Pt}$ segments, and $\mathrm{Ni}$ concentration was estimated using energy-dispersive $\mathrm{x}$-ray spectroscopy. More details about fabrication and magnetic properties of $\mathrm{Pt}_{1-x} \mathrm{Ni}_{x}$ films can be found in Refs. [43,44] and in Appendices $\mathrm{C}$ and $\mathrm{D}$. Nb/Ni/Nb JJ's with different $d_{F}$ were fabricated from the same wafer with a calibrated Ni-thickness gradient [24]. $\mathrm{Nb}(\mathrm{S})$ electrodes were $\sim 200 \mathrm{~nm}$ thick. Multilayers were first patterned by photolithography and reactive ion etching and then processed by focused ion beam (FIB) Nanoscale JJ's with sizes down to $\sim 60 \mathrm{~nm}$ were made by FIB nanosculpturing $[26,36,45]$. Small sizes are necessary both for achieving the monodomain state $[41,46]$ and for enhancing normal resistances to comfortably measurable values, $R_{n} \gtrsim$ $0.1 \Omega$. We present data for JJ's with different sizes, interlayer thicknesses, $d_{F}$, and compositions. Junction parameters are listed in Tables I-III of the Appendix. Properties of Josephson spin valves with similar $\mathrm{CuNi}$ and $\mathrm{Ni}$ interlayers can be found in Refs. [36] and [41]. Figure 1 shows (a) a scanning electron microscope (SEM) image of one of the studied $\mathrm{Nb} / \mathrm{Ni} / \mathrm{Nb}$ JJ's and (b) a sketch with a current path.

Measurements are performed in ${ }^{3} \mathrm{He}$ and ${ }^{4} \mathrm{He}$ closed-cycle cryostats. Magnetic field, parallel to the junction plane, is supplied by a superconducting solenoid. We will show measurements with field oriented either parallel $H_{\|}$(easy axis) or perpendicular $H_{\perp}$ (hard axis) to the long side of the JJ.

\section{RESULTS AND DISCUSSION}

Figures 1(c) and 1(d) show current-voltage characteristics $(I-V)$ at zero field for (c) $\mathrm{Nb} / \mathrm{Cu}_{0.4} \mathrm{Ni}_{0.6} / \mathrm{Nb}$ junction with 
TABLE II. Parameters of junctions with Pt and paramagnetic PtNi interlayers.

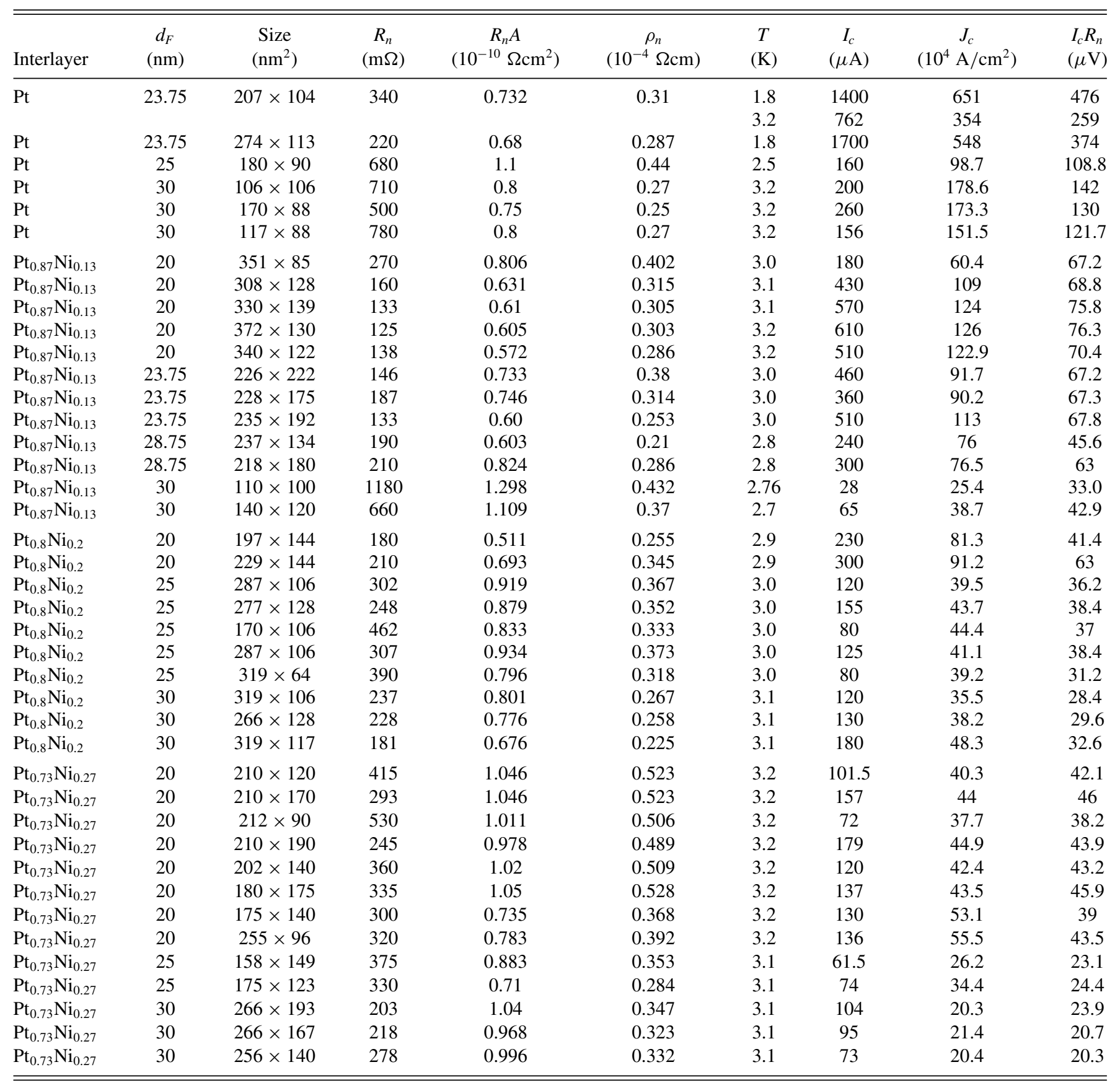

$d_{F}=10 \mathrm{~nm}$ at $T \simeq 0.5 \mathrm{~K}$ and $(\mathrm{d}) \mathrm{Nb} / \mathrm{Ni} / \mathrm{Nb}$ junction with $d_{F}=7 \mathrm{~nm}$ at different temperatures, $T=1.9-4.5 \mathrm{~K}$. The shapes of $I-V$ 's are typical for proximity-coupled $\mathrm{JJ}$ 's, described by the resistively shunted junction model.

Figures 1(e) and 1(f) show temperature dependencies of critical current densities for (e) the same $\mathrm{Nb} / \mathrm{Cu}_{0.4} \mathrm{Ni}_{0.6} / \mathrm{Nb}$ $\mathrm{JJ}$ and (f) $\mathrm{Nb} / \mathrm{Ni} / \mathrm{Nb} \mathrm{JJ}$ 's with $d_{F}=5,7,10$, and $20 \mathrm{~nm}$. It is seen that the $\mathrm{JJ}$ with a diluted $\mathrm{Cu}_{0.4} \mathrm{Ni}_{0.6}$ interlayer has a significantly smaller $J_{c}$ than the $\mathrm{JJ}$ with pure $\mathrm{Ni}$ with the same $d_{F}=10 \mathrm{~nm}$, compare red lines in Figs. 1(e) and 1(f). It is also seen that the $\mathrm{Cu}_{0.4} \mathrm{Ni}_{0.6} \mathrm{JJ}$ exhibits stronger superlinear temperature dependence with a positive curvature $d^{2} J_{c} / d T^{2}>0$ at elevated $T$, which is well described by the power-law dependence $J_{c} \propto\left(1-T / T_{c}\right)^{a}$ with $a \simeq 3.5$. On the other hand, Ni JJ's show almost linear $J_{c}(T)$, irrespective of $\mathrm{Ni}$ thickness, albeit with a varying onset temperature $T_{c}^{*}$.

\section{A. In situ magnetic characterization of $\mathrm{Ni}$ interlayers via absolute Josephson fluxometry}

Top panels in Fig. 2 represent measured $I_{c}(H)$ modulation patterns for $\mathrm{Nb} / \mathrm{Ni} / \mathrm{Nb} J J$ 's with different $d_{\mathrm{Ni}}$ (a) $5 \mathrm{~nm}$, (b) $7 \mathrm{~nm}$, (c) $10 \mathrm{~nm}$, and (d) $20 \mathrm{~nm}$. Junction sizes and measurement temperatures are indicated in the figure. Modulation patterns are shown both for easy (a),(b) and hard (c),(d) axis orientations. Blue and red lines represent up and down field sweeps. A hysteresis is due to finite coercivity of 
TABLE III. Parameters of junctions with ferromagnetic PtNi interlayers.

\begin{tabular}{|c|c|c|c|c|c|c|c|c|c|}
\hline Interlayer & $\begin{array}{c}d_{F} \\
(\mathrm{~nm})\end{array}$ & $\begin{array}{l}\text { Size } \\
\left(\mathrm{nm}^{2}\right)\end{array}$ & $\begin{array}{c}R_{n} \\
(\mathrm{~m} \Omega)\end{array}$ & $\begin{array}{c}R_{n} A \\
\left(10^{-10} \Omega \mathrm{cm}^{2}\right)\end{array}$ & $\begin{array}{c}\rho_{n} \\
\left(10^{-4} \Omega \mathrm{cm}\right)\end{array}$ & $\begin{array}{c}T \\
(\mathrm{~K})\end{array}$ & $\begin{array}{c}I_{c} \\
(\mu \mathrm{A})\end{array}$ & $\begin{array}{c}J_{c} \\
\left(10^{4} \mathrm{~A} / \mathrm{cm}^{2}\right)\end{array}$ & $\begin{array}{l}I_{c} R_{n} \\
(\mu \mathrm{V})\end{array}$ \\
\hline $\mathrm{Pt}_{0.6} \mathrm{Ni}_{0.4}$ & 25 & $630 \times 230$ & 50 & 0.725 & 0.29 & 2.2 & 160 & 11 & 8 \\
\hline $\mathrm{Pt}_{0.6} \mathrm{Ni}_{0.4}$ & 25 & $770 \times 320$ & 220 & 5.42 & 2.17 & 2.2 & 2070 & 84 & $455^{\mathrm{a}}$ \\
\hline $\mathrm{Pt}_{0.6} \mathrm{Ni}_{0.4}$ & 30 & $300 \times 165$ & 2000 & 9.9 & 3.3 & 3.0 & 140 & 28.3 & $280^{\mathrm{a}}$ \\
\hline $\mathrm{Pt}_{0.6} \mathrm{Ni}_{0.4}$ & 30 & $380 \times 180$ & 1030 & 5.19 & 1.73 & 3.0 & 7.5 & 1.5 & 7.7 \\
\hline $\mathrm{Pt}_{0.6} \mathrm{Ni}_{0.4}$ & 30 & $640 \times 225$ & 120 & 1.73 & 0.58 & 3.3 & 0 & 0 & 0 \\
\hline $\mathrm{Pt}_{0.6} \mathrm{Ni}_{0.4}$ & 30 & $640 \times 225$ & 300 & 4.32 & 1.44 & 2.8 & 110 & 7.6 & 33 \\
\hline \multirow[t]{2}{*}{$\mathrm{Pt}_{0.46} \mathrm{Ni}_{0.54}$} & 20 & $800 \times 225$ & 50 & 0.9 & 0.45 & 1.8 & 63 & 3.5 & 3.2 \\
\hline & & & & & & 3.0 & 28 & 1.56 & 1.4 \\
\hline \multirow[t]{2}{*}{$\mathrm{Pt}_{0.46} \mathrm{Ni}_{0.54}$} & 20 & $1140 \times 230$ & 120 & 3.15 & 1.57 & 1.8 & 2100 & 80 & $252^{\mathrm{a}}$ \\
\hline & & & & & & 3.0 & 800 & 30.5 & 96 \\
\hline \multirow[t]{2}{*}{$\mathrm{Pt}_{0.4} \mathrm{Ni}_{0.6}$} & 20 & $1050 \times 420$ & 25 & 1.1 & 0.55 & 1.8 & 136 & 3.08 & 3.4 \\
\hline & & & & & & 3.0 & 62 & 1.41 & 1.6 \\
\hline $\mathrm{Pt}_{0.4} \mathrm{Ni}_{0.6}$ & 25 & $630 \times 340$ & 51 & 1.09 & 0.44 & 0.4 & 81 & 3.8 & 4.1 \\
\hline $\mathrm{Pt}_{0.4} \mathrm{Ni}_{0.6}$ & 25 & $640 \times 340$ & 53 & 1.15 & 0.46 & 0.4 & 73 & 3.4 & 3.9 \\
\hline $\mathrm{Pt}_{0.4} \mathrm{Ni}_{0.6}$ & 25 & $670 \times 310$ & 53 & 1.10 & 0.44 & 0.4 & 58 & 2.8 & 3.1 \\
\hline $\mathrm{Pt}_{0.4} \mathrm{Ni}_{0.6}$ & 25 & $510 \times 310$ & 73 & 1.16 & 0.46 & 0.4 & 73 & 4.6 & 5.3 \\
\hline $\mathrm{Pt}_{0.4} \mathrm{Ni}_{0.6}$ & 25 & $550 \times 330$ & 71 & 1.29 & 0.52 & 0.4 & 91 & 5.0 & 6.5 \\
\hline $\mathrm{Pt}_{0.4} \mathrm{Ni}_{0.6}$ & 25 & $460 \times 300$ & 86 & 1.19 & 0.47 & 0.4 & 110 & 8.0 & 9.5 \\
\hline $\mathrm{Pt}_{0.4} \mathrm{Ni}_{0.6}$ & 25 & $560 \times 310$ & 69 & 1.2 & 0.48 & 0.4 & 49 & 2.8 & 3.4 \\
\hline $\mathrm{Pt}_{0.33} \mathrm{Ni}_{0.67}$ & 20 & $350 \times 190$ & 160 & 1.064 & 0.53 & 2.0 & 0 & 0 & 0 \\
\hline $\mathrm{Pt}_{0.33} \mathrm{Ni}_{0.67}$ & 20 & $350 \times 120$ & 260 & 1.092 & 0.55 & 2.0 & 0 & 0 & 0 \\
\hline
\end{tabular}

a The very large $I_{c} R_{n}$ values are not confident because the corresponding large $I_{c}$ is comparable to the onset of the flux-flow phenomenon in Nb electrodes. This leads to the nonlinear $I-V$ 's at large bias and makes it difficult to correctly estimate $R_{n}$.

F interlayers. It disappears at $H \sim \pm(1-1.5) \mathrm{kOe}$, corresponding to transition into the saturated magnetic state. All JJ's, included in the analysis, exhibit Fraunhofer-type $I_{c}(H)$ modulation, indicating good uniformity of interlayers [47]. Examples of $I_{c}(H)$ patterns for $\mathrm{Nb} / \mathrm{PtNi} / \mathrm{Nb}$ and $\mathrm{Nb} / \mathrm{Ni} / \mathrm{Nb}$ junctions can be found in Refs. [45] and [41], respectively.

The $I_{c}(H)$ modulation occurs due to flux quantization. This can be used for in situ AJF analysis [32,36,41], presented in middle panels of Fig. 2. Here symbols represent the flux $\Phi(H)$ at maxima and minima of $I_{c}(H)$, which correspond to half-integer and integer number of the flux quantum $\Phi_{0}$, respectively. The total flux is:

$$
\Phi=B L \Lambda+4 \pi M_{F} L d_{F},
$$

where $B$ is magnetic induction, $L$ is the junction length, $\Lambda$ is the effective magnetic thickness of the $\mathrm{JJ}$, and $M_{F}$ is magnetization of the F layer along the field. The first term in the right-hand side represents the flux induced by magnetic field; the second represents magnetization of the $\mathrm{F}$ layer (for more details see Appendix E).
From Figs. 2(a) and 2(b) it is seen that at large fields $\Phi(H)$ is linear. Since in this case F layers are in the saturated state, $M_{F}=M_{\text {sat }}$, the linear field dependence is caused solely by the first term in Eq. (1) with $B \propto H$. Subtraction of this linear dependence, shown by dashed lines in the middle panels of Fig. 2, reveals the contribution $\Delta \Phi$ from the second term in Eq. (1). This yields the absolute value of magnetization in the F interlayer $4 \pi M_{F}=\Delta \Phi / L d_{F}$. Thus obtained magnetization curves, $4 \pi M_{\mathrm{Ni}}(H)$, are shown in the bottom panels of Fig. 2. Saturation magnetizations are $4 \pi M_{\text {sat }}=8.2 \pm 1.1 \mathrm{kG}$ for $d_{\mathrm{Ni}}=5 \mathrm{~nm}, 7.3 \pm 0.5 \mathrm{kG}$ for $d_{\mathrm{Ni}}=7 \mathrm{~nm}$, and $6.9 \pm 0.3 \mathrm{kG}$ for $d_{\mathrm{Ni}}=10 \mathrm{~nm}$. For $d_{\mathrm{Ni}}=$ $20 \mathrm{~nm}$ the saturation magnetization is not reached within the shown field range (see Appendix E for clarifications). The main uncertainty in $M_{\text {sat }}$ is caused by the accuracy of estimation of $d_{\mathrm{Ni}}$, limited by the film roughness $R_{q} \simeq 1 \mathrm{~nm}$ (see Appendix A). The thinner the film, the larger such systematic uncertainty.

The obtained saturation magnetization $4 \pi M_{\text {sat }} \simeq 7 \mathrm{kG}$ is consistent with that for bulk nickel $[48,49]$. This implies that 


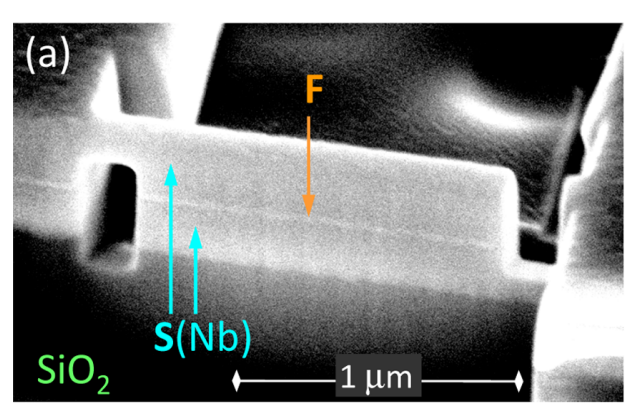

(b)

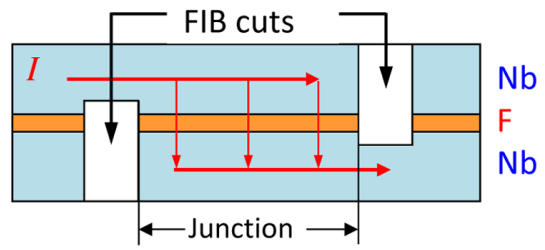

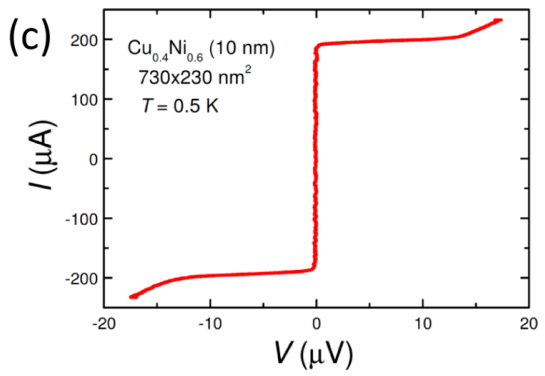

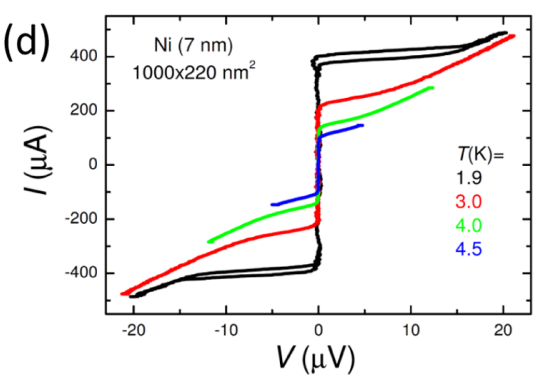

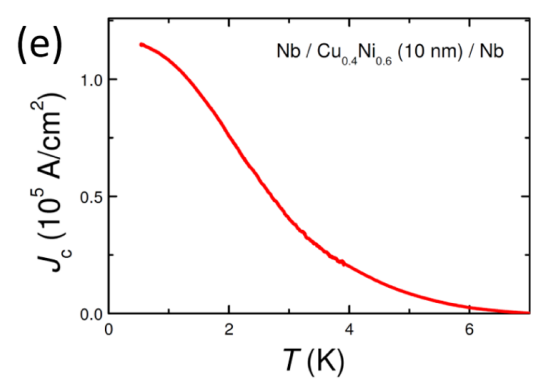

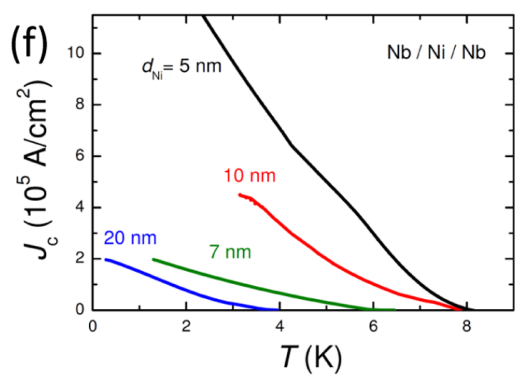

FIG. 1. (a) SEM image of an SFS junction and (b) a sketch with indication of current flow paths. (c) $\mathrm{The} I-V$ curve of $\mathrm{Nb} / \mathrm{Cu}{ }_{0.4} \mathrm{Ni} i_{0.6} / \mathrm{Nb}$ junction with $d_{F}=10 \mathrm{~nm}$. (d) A set of $I-V$ 's of a $\mathrm{Nb} / \mathrm{Ni}(7 \mathrm{~nm}) / \mathrm{Nb}$ junction at different $T$. (e),(f) Temperature dependencies of the critical current density for (e) the same $\mathrm{Nb} / \mathrm{Cu}_{0.4} \mathrm{Ni}_{0.6} / \mathrm{Nb}$ junction and (f) $\mathrm{Nb} / \mathrm{Ni} / \mathrm{Nb}$ junctions with different $\mathrm{Ni}$ thicknesses of $5,7,10$, and $20 \mathrm{~nm}$. Note that the $J_{c}(T=3 \mathrm{~K})$ for the $\mathrm{Ni}$ junction, $d_{F}=10 \mathrm{~nm}$, is almost an order of magnitude larger than that for the junction with diluted $\mathrm{Cu}_{0.4} \mathrm{Ni}_{0.6}$ interlayer with the same $d_{F}$.

$\mathrm{Ni}$ interlayers in our JJ's are fully ferromagnetic and there are no extended dead magnetic layers, i.e., interface layers of $\mathrm{Ni}$ with reduced magnetism. Such dead layers, accompanied by a significant reduction of $M_{\text {sat }}$, were reported in earlier works $[23,26]$ and would make interpretation of the proximity effect more complicated. On the other hand, a variation of the superconducting onset temperature, which can be seen in Figs. 1(f) and 3(b), provides evidence for the existence of dead superconducting (rather than magnetic) layers with suppressed $T_{c}^{*}$ at junction interfaces.
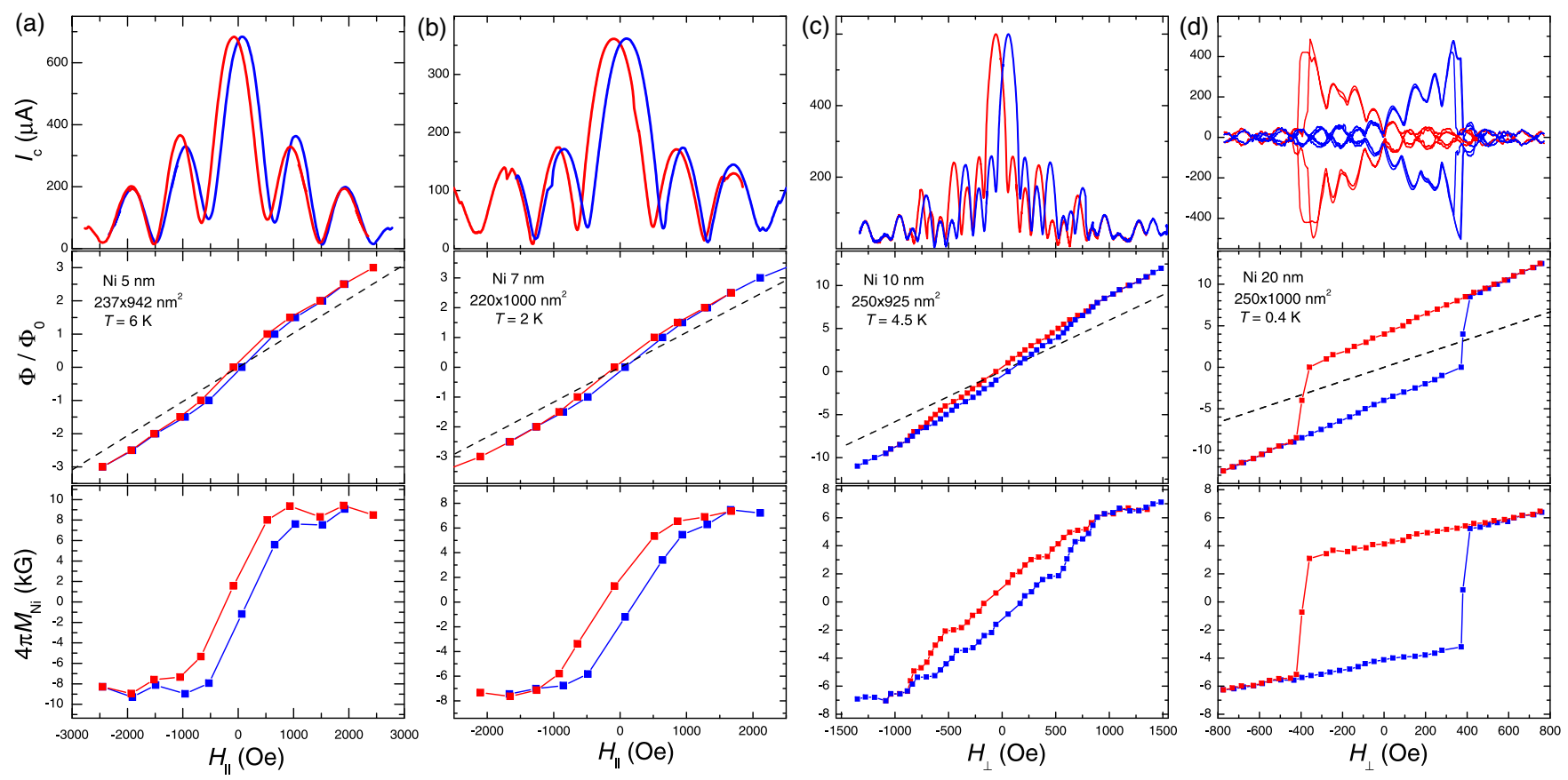

FIG. 2. Top panels: magnetic field modulation of the critical current, $I_{c}(H)$, for Nb/Ni/Nb JJ's with $d_{\mathrm{Ni}}$ (a) $5 \mathrm{~nm}$ and (b) $7 \mathrm{~nm}$ in the easy axis orientation and (c) $10 \mathrm{~nm}$ and (d) $20 \mathrm{~nm}$ in the hard axis orientation. Blue/red lines represent up/down field sweeps. Middle panels show the absolute Josephson fluxometry analysis of the data above. Symbols represent positions of maxima and minima of the $I_{c}(H)$ patterns, which correspond to half-integer and integer values of $\Phi / \Phi_{0}$. Bottom panels represent magnetization curves of Ni interlayers obtained from the AJF analysis. Large values of the saturation magnetization $4 \pi M_{\mathrm{Ni}} \simeq 7 \mathrm{kG}$ are consistent with the value for bulk Ni and preclude the presence of dead magnetic layers. 

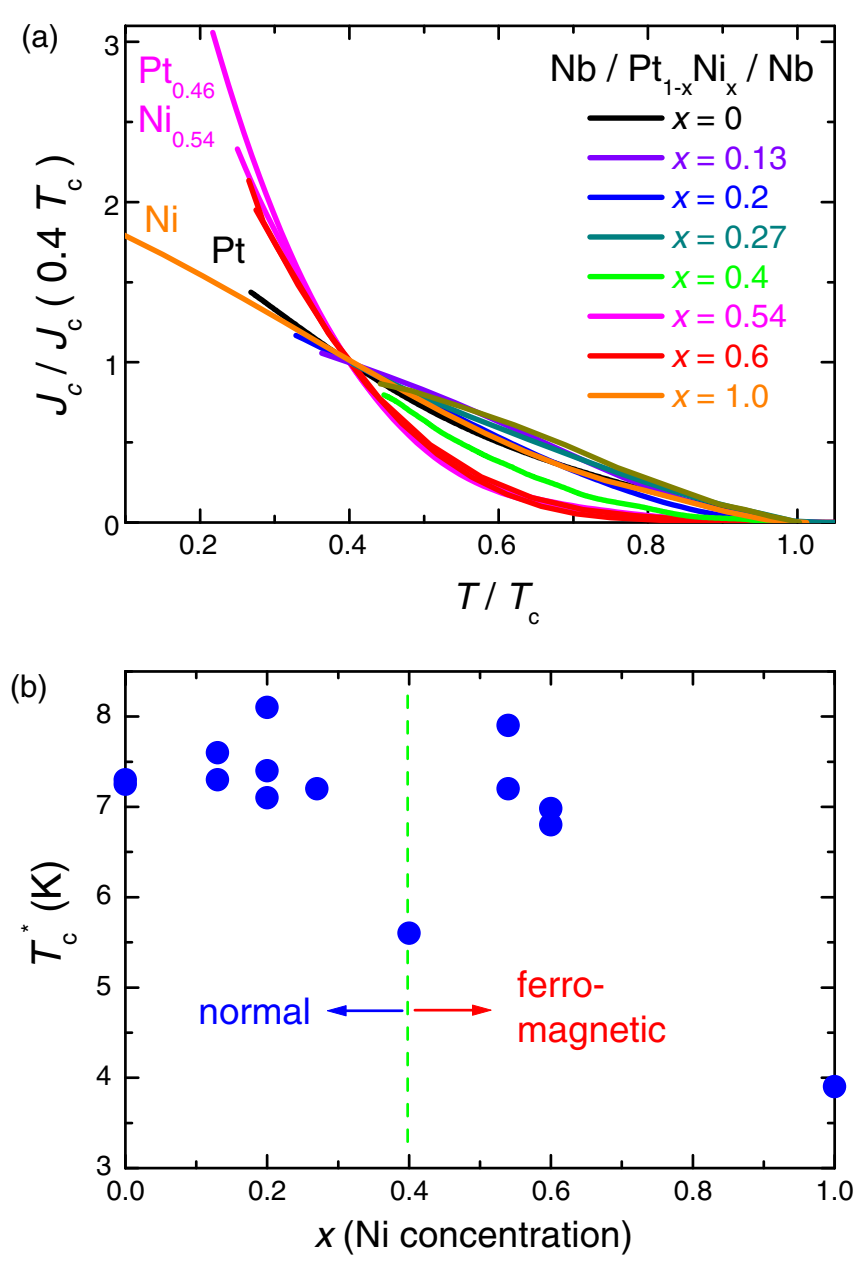

FIG. 3. (a) Temperature dependencies $J_{c}(T)$ of $\mathrm{Nb} / \mathrm{Pt}_{1-x} \mathrm{Ni}_{x} / \mathrm{Nb}$ junctions with $d_{F}=20 \mathrm{~nm}$, normalized to the value at $T=0.4$ of the onset temperature. A gradual evolution of $J_{c}(T)$ dependencies from linear for pure Pt, $x=0$, to superlinear at $x \simeq 0.5-0.6$ and back to linear for pure $\mathrm{Ni}, x=1$, indicates a transition from SNS to dirty SFS and to clean SFS cases. (b) Corresponding onset temperatures of the junctions versus $\mathrm{Ni}$ concentration.

The large value of $M_{\text {sat }}$ confirms that supercurrents in our $\mathrm{Nb} / \mathrm{Ni} / \mathrm{Nb}$ JJ's flow through a pure $\mathrm{Ni}$ with strong ferromagnetic properties. Remarkably, we observe a large $J_{c} \simeq$ $2 \times 10^{5} \mathrm{~A} / \mathrm{cm}^{2}$, even through $20 \mathrm{~nm}$ of $\mathrm{Ni}$, see Fig. 1(f). This is a much longer scale compared to earlier reports $[21,26,27,31,34]$ in which supercurrent was observed only through a few nm of Ni. Observation and clarification of such a profound LRPE through a strong $F$ is the main objective of this work.

\section{B. Temperature dependencies of critical currents: Crossover between dirty and clean regimes}

To clarify our observations, we start with a short summary of proximity effects in SNS and SFS JJ's (more detailed analysis can be found in Ref. [50], where various regimes have been considered). In SNS JJ's $J_{c}$ is determined by the superconducting order parameter at the junction interface $\Psi_{S / N}$ and the ratio of the thickness $d_{N}$ to the coherence length $\xi_{N}$ of the interlayer. Close to $T_{c}$ it can be written in the following simple form [51],

$$
J_{c} \propto \Psi_{S / N}^{2} \exp \left(-\frac{d_{N}}{\xi_{N}}\right) .
$$

For JJ's with thick $d_{N}$, or short $\xi_{N}, d_{N} \gg \xi_{N}\left(T_{c}\right)$, the $J_{c}(T)$ is determined predominantly by the $T$ dependence of $\xi_{N}(T)$, leading to a strong superlinear $T$ dependence. In the opposite case, $d_{N} \ll \xi_{N}\left(T_{c}\right), J_{c}(T)$ is determined by $\Psi_{S / N}(T)$, leading to a conventional linear $J_{c}(T)$ close to $T_{c}$ and a saturation at $T \rightarrow 0$.

For SFS JJ's the coherence length $\xi_{F}$ is complex $[6,20]$,

$$
\xi_{F}^{-1}=\xi_{F 1}^{-1}+i \xi_{F 2}^{-1} .
$$

The real part, $\xi_{F 1}$, represents the decay length; the imaginary, $\pi \xi_{F 2}$, represents the period of oscillations. In the clean case,

$$
\xi_{F}(c)=\frac{\hbar v_{f}}{2\left(\pi k_{B} T+i E_{\mathrm{ex}}\right)},
$$

where $v_{f}$ is the Fermi velocity in F. In the dirty case,

$$
\xi_{F}(d)=\sqrt{\frac{l_{e}}{3} \xi_{F}(c) .}
$$

Since here $l_{e} \ll\left|\xi_{F}(c)\right|$, the coherence length in the dirty case is shorter than in the clean case. For strong $\mathrm{F}$ with $E_{\mathrm{ex}} / k_{B} \gg$ $T_{c}$, in the dirty case $\xi_{F 1}(d) \simeq \xi_{F 2}(d) \simeq\left(\hbar l_{e} v_{f} / 3 E_{\mathrm{ex}}\right)^{1 / 2}$ are equally short. However, in the clean case the two scales are different: $\xi_{F 1}(c) \simeq\left(\hbar v_{f} / 2 \pi k_{B} T\right)$ is as long as $\xi_{N}$, and $\xi_{F 2}(c) \simeq\left(\hbar v_{f} / 2 E_{\mathrm{ex}}\right)$ is short $[8,14,15,24]$. From the discussion above, it follows that the shape of $J_{c}(T)$ provides an important clue about the proximity effect $[20,22,23,26]$.

Figure 3(a) shows the evolution of $J_{c}(T)$, normalized to the value at $0.4 T_{c}^{*}$ for $\mathrm{Nb} / \mathrm{Pt}_{1-x} \mathrm{Ni}_{x} / \mathrm{Nb} \mathrm{JJ}$ 's with different $\mathrm{Ni}$ concentrations and $d_{F} \simeq 20 \mathrm{~nm}$. The onset temperature $T_{c}^{*}$ is shown in Fig. 3(b). Ferromagnetism in $\mathrm{Pt}_{1-x} \mathrm{Ni}_{x}$ appears at a critical concentration $x_{c}=0.4[43,44]$, as described in Appendix C. In Fig. 3(b), apart from a minimum of $T_{c}^{*}$ for the pure $\mathrm{Ni}, x=1$, we can also see a clear minimum at $x_{c}=0.4$. Both minima can be interpreted as being due to suppression of $\Psi_{S / N}$ at the interface (dead superconducting layer) due to either a reverse magnetic proximity effect for pure $\mathrm{Ni}, x=1$, or quantum fluctuations at the quantum critical point, $x_{c}=0.4$ [44].

From Fig. 3(a) it can be seen that for a pure Pt, $x=0$ (black line), $J_{c}(T)$ is almost linear. As explained above, this is expected for SNS JJ's with $\xi_{N}\left(T_{c}\right) \gg d_{N}$. Upon increasing Ni concentration, $J_{c}(T)$ remains linear for nonmagnetic interlayers $x=0.13,0.2,0.27$. At the critical concentration, $x_{c}=0.4$ (green line), a positive curvature develops in $J_{c}(T)$. It becomes most pronounced for $x=0.5-0.6$ (magenta and red curves). Such a transformation is the consequence of a rapid reduction of $\xi_{F}$ both due to enhancement of $E_{\mathrm{ex}}$ and reduction of $l_{e}$. While $E_{\mathrm{ex}}$ increases linearly at $x>x_{c}$, the m.f.p. reaches minimum at $x \simeq 0.5$, corresponding to atomically disordered, $l_{e} \sim 1 \mathrm{~nm}$ [14], dirty metal (see Appendix C). With further increase of concentration to pure $\mathrm{Ni}, x=1$ (orange line), the linear $J_{c}(T)$ dependence is restored, similar to a pure $\mathrm{Pt}$, $x=0$. Such a recovery implies that $\xi_{F 1}$ in pure $\mathrm{Ni}$ is similarly long as $\xi_{N}$ for pure $\mathrm{Pt}$, despite the large $E_{\mathrm{ex}} \sim 10^{3} \mathrm{~K}$. As discussed above, this indicates occurrence of clean, ballistic 
transport in Ni. Thus, variation of the shape of $J_{c}(T)$ reveals two crossovers in electron transport regimes with changing $\mathrm{Ni}$ concentration. First a crossover takes place from a clean SNS type proximity effect in pure Pt to a dirty SFS case in diluted, atomically disordered alloy $x \simeq 0.5$. With further increase of $x$ a second crossover from dirty to clean ballistic transport takes place for JJ's with pure Ni.

As mentioned in the Introduction, interpretation of LRPE in strong ferromagnets is still controversial. In several cases it was attributed to appearance of the unconventional oddfrequency spin-triplet order parameter. Recently the dominant $(\sim 70 \%)$ spin-triplet supercurrent was reported in $\mathrm{SF}_{1} \mathrm{~F}_{2} \mathrm{~S}$ Josephson spin-valve structures with similar $\mathrm{Ni}$ interlayers [41]. However, the triplet supercurrent appears only in the noncollinear state of the spin valve and is tuned by the relative orientation of magnetization in the two F layers. Appearance and disappearance of the long-range triplet supercurrent upon remagnetization of the spin valve leads to a profound distortion of the $I_{c}(H)$ pattern [41]. Such a distortion is the main fingerprint of the triplet component [46] and, thus, provides the key evidence for it's existence. SFS junctions, containing just a single $\mathrm{F}$ layer, behave completely differently (see, e.g., the discussion in Sec. IV C of Ref. [41]). In particular, $I_{c}(H)$ patterns of all our junctions are Fraunhofer-like, with the only distortion caused by the hysteresis in $M_{F}(H)$. As discussed in the Introduction, the triplet state is not anticipated in SFS junctions because there is no obvious mechanism for appearance of the noncollinear magnetic state in the perpendicular direction across the single F-layer. Therefore, we want to emphasize that LRPE in $\mathrm{Nb} / \mathrm{Ni} / \mathrm{Nb} J J$ 's with clean $\mathrm{Ni}$ is achieved by the spin-singlet current without involvement of the unconventional odd-frequency spin-triplet order parameter. Such LRPE is simply a consequence of the lack of scattering mechanism that can destroy singlet Cooper pair correlations in a clean metal (no matter $\mathrm{F}$ or $\mathrm{N}$ ) at $T=0$ [12]. Thus, it is the cleanliness of pure $\mathrm{Ni}$ that facilitates LRPE in $\mathrm{Nb} / \mathrm{Ni} / \mathrm{Nb}$ JJ's. Concurrently, the extreme dirtiness suppresses proximity effect through diluted $\mathrm{F}$ alloys, despite a small $E_{\text {ex }}$.

We also studied $\mathrm{Nb} / \mathrm{Cu}(10 \mathrm{~nm}) / \mathrm{Ni}(10 \mathrm{~nm}) / \mathrm{Nb}$ JJ's, with $\mathrm{Cu} / \mathrm{Ni}$ bilayer. Interestingly, they show an order of magnitude smaller $J_{c}$ than $\mathrm{Nb} / \mathrm{Ni}(10 \mathrm{~nm}) / \mathrm{Nb} J$ J's, see Table I in the Appendix, consistent with earlier results for Ni-based JJ's with $\mathrm{Cu}$ buffer layers [27,31,34]. This is surprising because, due to a large $\xi_{N} \sim 1 \mu \mathrm{m}$ of $\mathrm{Cu}, 10 \mathrm{~nm}$ should have little influence. On the other hand, neighbors in the periodic table $\mathrm{Cu}$ and $\mathrm{Ni}$ tend to easily alloy with each other. Therefore, $\mathrm{Cu} / \mathrm{Ni}$ bilayers likely contain a dirty $\mathrm{CuNi}$ interlayer, which leads to suppression of $J_{c}$.

\section{CONCLUSIONS}

To conclude, we have studied SFS junctions with different Ni-based interlayers. We observed that supercurrents through pure Ni may be much larger than through diluted alloys with much smaller $E_{\text {ex }}$. Analysis of $J_{c}(T)$ dependencies revealed that this counterintuitive result is caused by the dirtiness of disordered $\mathrm{Ni}$ alloys, leading to a short coherence lengths $\xi_{F 1} \sim 1 \mathrm{~nm}$. To the contrary, the mean-free path in pure $\mathrm{Ni}$ interlayers can easily exceed the film thickness [52] up to several tens of nm, facilitating ballistic Cooper pair transport with the decay length as long as in nonmagnetic normal metals. Our observation suggests that SFS junctions with strong-but-clean ferromagnets may have significant advantages, compared to commonly considered weak-but-dirty alloys.

Our results may also help to resolve the controversy around LRPE in strong ferromagnets, which is either seen $[19,30,35]$ or not $[21,40]$. We want to emphasize that proximity effect in ferromagnets essentially depends on the internal structure. In contrast to SNS JJ's, which always show LRPE at low enough temperatures because $\xi_{N}(T \rightarrow 0) \rightarrow \infty$ irrespective of cleanliness, for SFS JJ's LRPE occurs only in the clean case, for which $\xi_{F 1}(c) \rightarrow \infty$ at $T \rightarrow 0$, while for the dirty case $\xi_{F 1}(d)$ remains short irrespective of $T$. This leads to a principle difference in the range of proximity effects for clean and dirty ferromagnets with otherwise similar compositions and exchange energies.

\section{ACKNOWLEDGMENTS}

The work was supported by the EU H2020WIDESPREAD-05-2017-Twinning project "SPINTECH," Grant Agreement No. 810144 (sample preparation and measurements) and the Russian Science Foundation Grant No. 19-19-00594 (data analysis and manuscript preparation). The manuscript was written during a sabbatical semester of V.M.K. at MIPT, supported by the Faculty of Natural Sciences at SU.

\section{APPENDIX A: Nb/Ni FILM STRUCTURE}

Figure 4 shows topography maps obtained by atomic force microscopy for (a) a $\mathrm{Nb}$ film with thickness $d=100 \mathrm{~nm}$ and (b)-(d) $\mathrm{Nb} / \mathrm{Ni}$ bilayers with increasing Ni thickness. It can be seen that the $\mathrm{Nb}$ film has a rise-seed-like structure with elongated crystallites (a). In $\mathrm{Nb} / \mathrm{Ni}$ bilayers, with increasing $\mathrm{Ni}$ thickness $d_{\mathrm{Ni}}$, the structure of $\mathrm{Ni}$ first inherits that of $\mathrm{Nb}$ (b) but at $d_{\mathrm{Ni}} \simeq 5 \mathrm{~nm}$ a reconstruction to square-shaped crystallites occurs (c), which do not change significantly in shape and size ( $\sim 20 \mathrm{~nm}$ ) with further increase of $d_{\mathrm{Ni}}$ (in the studied range). The mean-square-root roughness of all films is $R_{q} \simeq 1 \mathrm{~nm}$, although few spikes up to $\sim \pm 4 \mathrm{~nm}$ can be seen in all cases. Probably because of that, we could not obtain reliable data for junctions with $d_{\mathrm{Ni}}<5 \mathrm{~nm}$, which were usually shorted and did not exhibit JJ behavior. As can be seen from Fig. 4, the overall roughness of the junction $\mathrm{S} / \mathrm{F}$ interface is determined by the roughness of the bottom $\mathrm{Nb}$ layer and is $R_{q} \simeq 1 \mathrm{~nm}$ for all studied JJ's.

\section{APPENDIX B: SUMMARY OF JUNCTION CHARACTERISTICS}

Tables I-III represent characteristics of all types of studied junctions. Figure 5 summarizes measured critical current densities at $T=3 \mathrm{~K}$ for JJ's with different interlayer composition and thickness, studied in this work. $J_{c}$ decreases both with increasing $\mathrm{Ni}$ concentration and interlayer thickness. For $\mathrm{Nb} / \mathrm{Ni} / \mathrm{Nb} J J$ 's (blue) we have sufficient samples to observe the nonmonotonous dependence $J_{c}$ vs $d_{F}$ due to $0-\pi$ transitions $[20,22,23,26]$. The blue line, connecting points for Ni-JJ's, however, is drawn solely for the easiness of identification of the data points and does not reflect the anticipated 
(a)

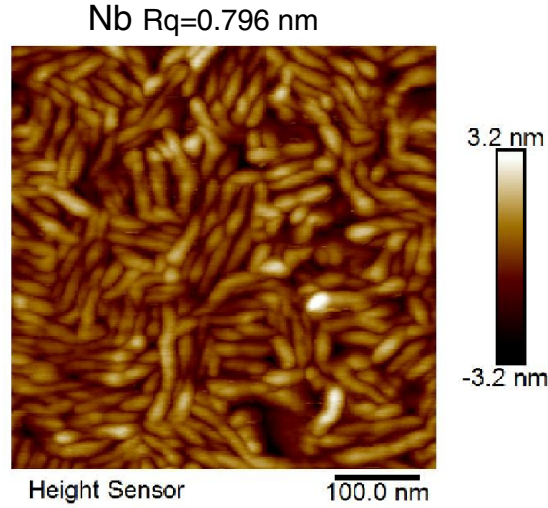

(c)

$$
\mathrm{Nb} / \mathrm{Ni}(6 \mathrm{~nm}) \mathrm{Rq}=1.22 \mathrm{~nm}
$$

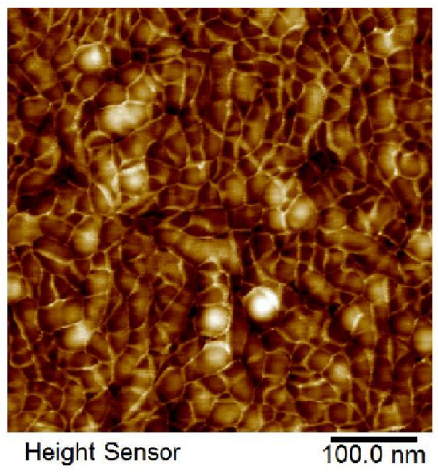

$\mathrm{Nb} / \mathrm{Ni}(2 \mathrm{~nm}) \mathrm{Rq}=1.18 \mathrm{~nm}$

(b)

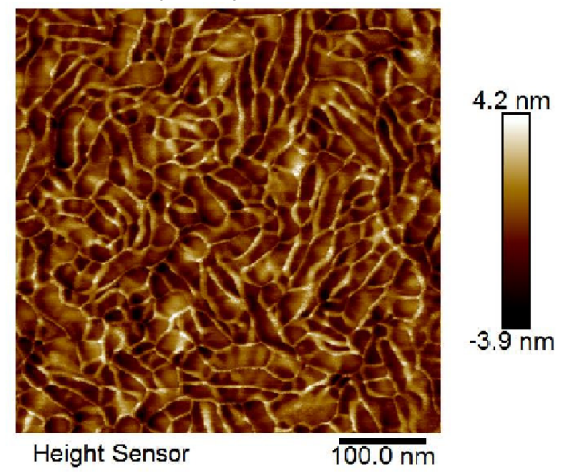

(d)

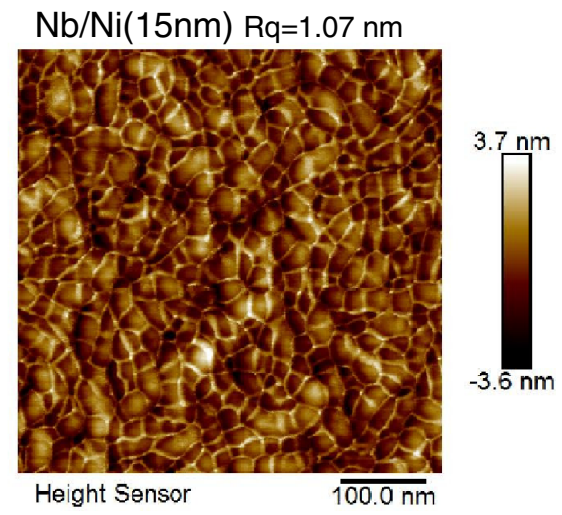

FIG. 4. Atomic force microscope topography maps of a $100 \mathrm{~nm}$ thick $\mathrm{Nb}$ film (a) without $\mathrm{Ni}$ on top, and (b) with $2 \mathrm{~nm} \mathrm{Ni,} \mathrm{(c)} 6 \mathrm{~nm} \mathrm{Ni}$, and (d) $15 \mathrm{~nm}$ Ni films on top. It can be seen that the Ni film reconstruction occurs at about $5 \mathrm{~nm}$ thickness.

$J_{c}\left(d_{F}\right)$ dependence, which should oscillate at a much shorter scale $\xi_{F 2}(\mathrm{Ni}) \sim 1 \mathrm{~nm}[26]$.

Our conclusions are based on the overall analysis of SFS junctions, as listed in Tables I and III. In particular we want

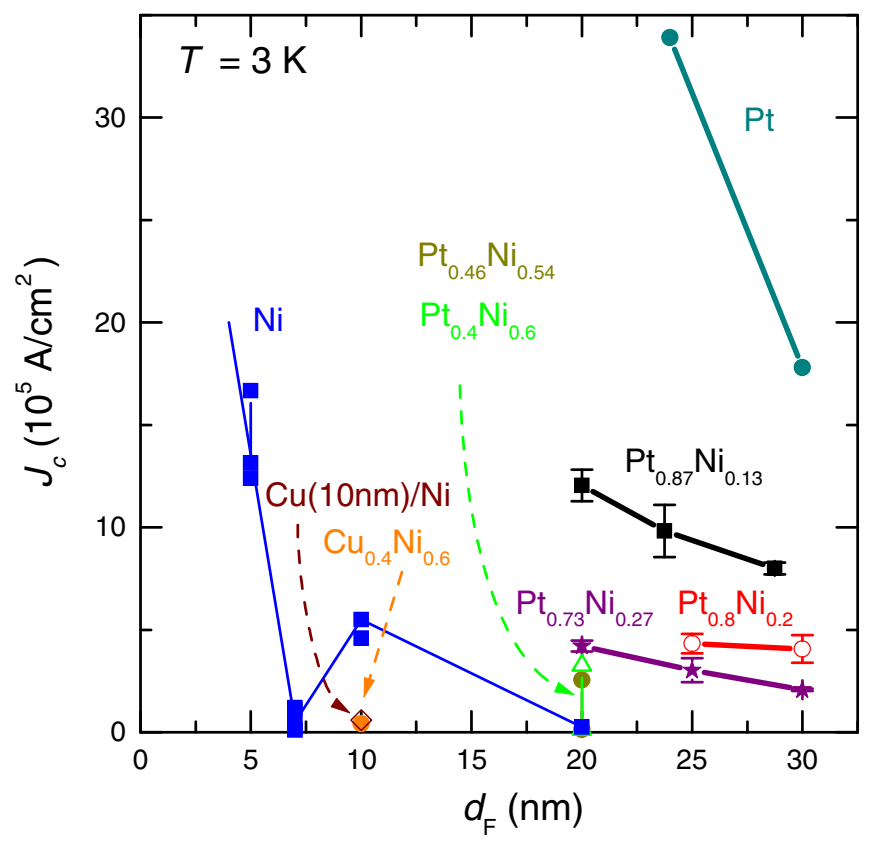

FIG. 5. A summary of measured critical current densities for different junctions at $T=3 \mathrm{~K}$ versus the interlayer thickness. to note that the small $J_{c}$ value for $\mathrm{Ni}(20 \mathrm{~nm}) \mathrm{JJ}$ in Fig. 5 is the consequence of the much lower onset temperature $T^{*}<4 \mathrm{~K}$ for this JJ, as shown in Fig. 3(b). The data in Fig. 5 is shown for $T=3 \mathrm{~K}$. This temperature was chosen because we have data for this $T$ for all junctions. The low $T^{*}$ of Ni-JJ results in the misleadingly low $J_{c}(3 \mathrm{~K})$, as can be seen from Fig. 1(f). More appropriate comparison should be done for $T \ll T^{*}$. Such data is listed in Tables I and III and is consistent with our conclusion. One should also keep in mind the oscillatory dependence of $J_{c}$ on $d_{F}$ with a nm-scale period of oscillations. Since the periods are different for different ferromagnets, it becomes impossible to make a conclusion by comparing just two JJ's with a fixed $d_{F}$. For the same reason we do not claim that $J_{c}$ in $\mathrm{Ni}$ is always larger than in an alloy (which cannot be true due to different oscillatory dependencies of the two). Also, because of that we cannot make an estimation of decay lengths for our SFS junction. Somewhat reliable decay length estimation from the data in Fig. 5 could be made only for nonmagnetic alloys with $x<0.4$ (see, e.g., black and violet lines in Fig. 5 and Table II) because such JJ's should not exhibit oscillations.

\section{APPENDIX C: PROPERTIES OF Nb/Pt $\mathrm{Pt}_{1-x} \mathrm{Ni}_{x} / \mathrm{Nb}$ JUNCTIONS}

Group-10 elements $\mathrm{Pt}$ and $\mathrm{Ni}$ are well intermixed with each other and can form solid solutions at arbitrary proportions [53-55]. PtNi alloys should be fairly uniform, contrary to $\mathrm{CuNi}$ alloys which are prone to phase segregation and 

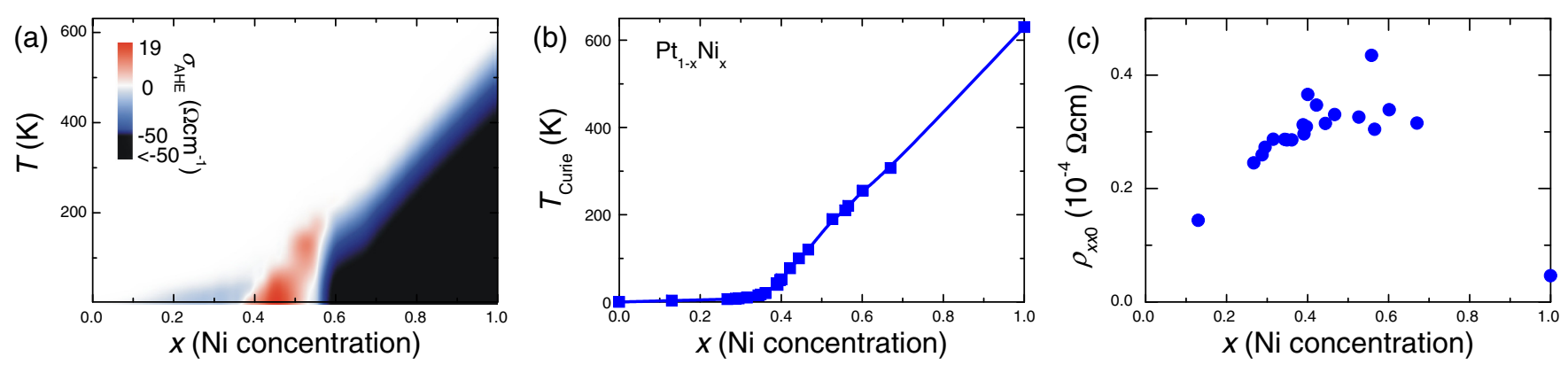

FIG. 6. Characteristics of $\mathrm{Pt}_{1-x} \mathrm{Ni}_{x}$ thin films. (a) Anomalous Hall effect conductivity versus temperature and Ni concentration. (b) Curie temperature obtained from the AHE data. Note that the magnetic quantum phase transition with $T_{\text {Curie }} \simeq 0$ occurs at $x_{c} \simeq 0.4$. (c) Residual in-plane resistivity of films. Maxima of $\rho_{x x}$ are observed at $x \simeq 0.4$ and 0.6 , corresponding to the sign-reversal points of the AHE in panel (a). Data from Refs. [43,44].

formation of Ni clusters. Therefore, we have chosen this alloy for detailed analysis of variation of properties of SFS junction with the strength of the $\mathrm{F}$ interlayer. For that we made a series of $\mathrm{Nb} / \mathrm{Pt}_{1-x} \mathrm{Ni}_{x} / \mathrm{Nb}$ JJ's with different $\mathrm{Ni}$ concentrations $x=0-1$. Composition of $\mathrm{Pt}_{1-x} \mathrm{Ni}_{x}$ films was estimated using energy-dispersive $\mathrm{x}$-ray spectroscopy [43].

Figures 6(a) and 6(b) summarize magnetic properties of thin $\mathrm{Pt}_{1-x} \mathrm{Ni}_{x}$ films (35-45 nm thick) obtained in earlier works $[43,44]$. Figure 6(a) shows the anomalous Hall effect (AHE) conductivity $\sigma_{x y}$ at the $T-x$ phase diagram. The AHE indicates the appearance of the ferromagnetic state [56]. Figure 6(b) shows the Curie temperature extracted from Hall measurements. It is seen that ferromagnetism in $\mathrm{Pt}_{1-x} \mathrm{Ni}_{x}$ thin films appears at $x>0.4$, similar to bulk alloys [54].

$\mathrm{Pt}_{1-x} \mathrm{Ni}_{x}$ alloys may form a disordered fcc state $(A 1)$, which is presumably dominant in our sputtered films. However there are also three ordered states $\mathrm{Pt}_{3} \mathrm{Ni}\left(L 1_{2}\right), \mathrm{PtNi}$ $\left(L 1_{0}\right)$, and $\mathrm{Ni}_{3} \mathrm{Pt}\left(L 1_{2}\right)$ with centra of stability at $x=0.25,0.5$, and 0.75 , respectively $[53,55]$. The most remarkable feature of the AHE in PtNi films, Fig. 6(a), is the sign change of $\sigma_{x y}$ from electronlike to holelike at $x \simeq 0.4-0.6$, which coincides with the expected range of stability of the layered $L 1_{0} \mathrm{PtNi}$ compound [53,55,57].

Figure 6(c) represents residual longitudinal resistivities of the films at $T=2 \mathrm{~K}$. The $\rho_{x x 0}$ increases upon mixing of $\mathrm{Ni}$ and $\mathrm{Pt}$ with a maximum around $x \simeq 0.5$. This indicates a progressive shortening of the electronic m.f.p. due to the growing disorder. The 50-50 mixture has almost an order of magnitude larger $\rho_{x x 0}$ than the pure Ni film $x=1$. Simultaneously we also see sharp peaks at $x \simeq 0.4$ and 0.6 , which indicates that additional frustrations in the film structure appear at the borders between stability regions of the ordered $L 1_{2}$ and $L 1_{0}$ states.

Since ferromagnetism in $\mathrm{Pt}_{1-x} \mathrm{Ni}_{x}$ alloy appears at $x_{c} \simeq$ 0.4 , diluted ferromagnets with small $T_{\text {Curie }} \sim 10 \mathrm{~K}$, comparable to $T_{c}$ of $\mathrm{Nb}$, correspond to an extremely dirty metallic state. The short electronic m.f.p. leads to a short $\xi_{F}$, which leads to a rapid suppression of the proximity induced superconducting order parameter with increasing $d_{F}[6,15]$. Therefore, as discussed in the paper, SFS junctions with weak disordered ferromagnets may have small critical current densities despite small exchange fields.

$\mathrm{Nb} / \mathrm{Pt}_{1-x} \mathrm{Ni}_{x} / \mathrm{Nb}$ junctions with different $x$ and $d_{F}$ were fabricated and studied, see Table II. An example of $I_{c}(H)$ modulation for $\mathrm{Nb} / \mathrm{PtNi} / \mathrm{Nb} \mathrm{JJ}$ can be found in Fig. 4 of Ref. [45]. Figure 7(a) shows measured $J_{c}(T=3 \mathrm{~K})$ for $\mathrm{Nb} / \mathrm{Pt}_{1-x} \mathrm{Ni}_{x} / \mathrm{Nb}$ junctions versus $\mathrm{Ni}$ concentration and
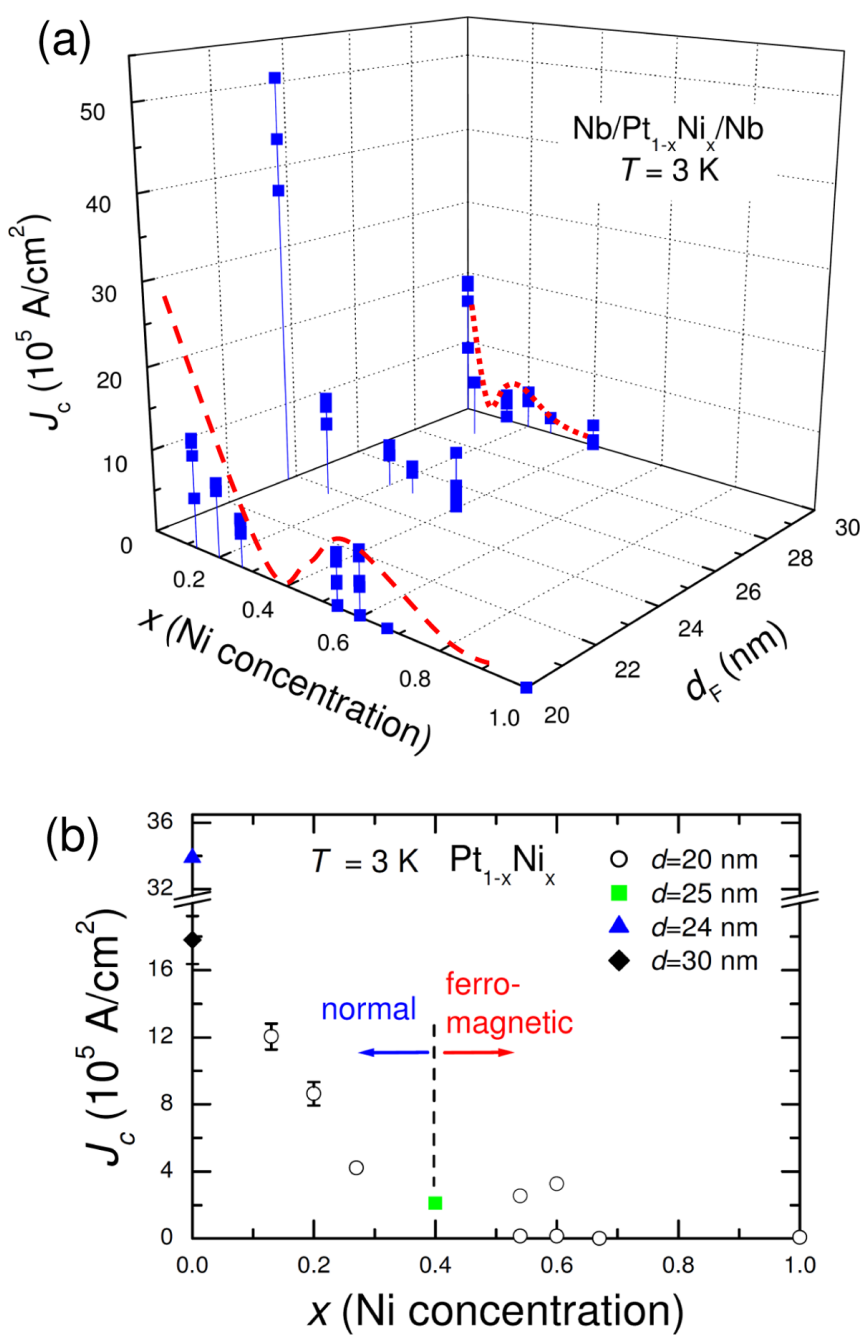

FIG. 7. (a) Three-dimensional plot of critical current densities of $\mathrm{Nb} / \mathrm{Pt}_{1-x} \mathrm{Ni}_{x} / \mathrm{Nb}$ junctions at $T=3 \mathrm{~K}$ as a function of $\mathrm{Ni}$ concentration and interlayer thickness. (b) Projection of the same data to the two-dimensional plot. Red dashed lines in (a) indicate possible $0-\pi$ transition for a fixed $d_{F}$ upon increasing Ni concentration. 
interlayer thickness. Figure 7(b) shows projection of this data to the $J_{c}-x$ plane. Generally, $J_{c}$ decreases both with increasing $x$ and $d_{F}$. However, it decreases nonmonotonously. Oscillatory decay of $J_{c}$ vs $d_{F}$ in SFS junctions is well documented and is caused by sequential $0-\pi$ transitions $[20,22,23,26]$.

From Fig. 7 it can be seen that for a given $d_{F}$ the $J_{c}$ is decaying nonmonotonously with increasing $\mathrm{Ni}$ concentration $x$, as indicated by dashed red lines in Fig. 7(a) for $d_{F}=20$ and $30 \mathrm{~nm}$. We attribute such oscillatory behavior to $0-\pi$ transitions at a given $d_{F}$ upon increasing the ferromagnetic exchange energy $E_{\text {ex }}$. The increase of Ni concentration leads to the enhancement of $E_{\mathrm{ex}}$, which leads to the shrinking of $\xi_{F}$ and causes the $0-\pi$ transition. As described above, ferromagnetism in $\mathrm{Pt}_{1-x} \mathrm{Ni}_{x}$ films appears at the critical concentration $x_{c} \simeq 0.4$. We observe that the relative spread in $J_{c}$ values increases in JJ's with the ferromagnetic interlayer $x>0.4$. Most likely this is also a consequence of a rapid shrinkage of $\xi_{F}$ down to about $1 \mathrm{~nm}$, comparable to the roughness of our films, see Fig. 4.

\section{APPENDIX D: INTERFACE RESISTANCES IN $\mathrm{Nb} / \mathrm{Pt}_{1-x} \mathrm{Ni}_{x} / \mathrm{Nb}$ JUNCTIONS}

Figure 8(a) shows a 3D plot of measured normal resistivities $\rho_{n}$ of studied $\mathrm{Nb} / \mathrm{Pt}_{1-x} \mathrm{Ni}_{x} / \mathrm{Nb}$ junctions versus $\mathrm{Ni}$ concentration and interlayer thickness. Parameters of JJ's are listed in Table III. Here $\rho_{n}=R_{n} A / d_{F}$, where $A$ is the junction area. Figure $8(\mathrm{~b})$ shows the $2 \mathrm{D}$ projection of the same data. It is seen that $\rho_{n}$ greatly increases at $x=0.4-0.6$ which is correlated with the region with maximal longitudinal resistance of PtNi films, see Fig. 6(c). Thus frustration and disorder is directly reflected in junction characteristics. However, $\rho_{n}$ exhibits much larger peaks at the frustration points $x \simeq 0.4$ and 0.6, compared to $\rho_{x x 0}$. Especially at the onset of ferromagnetism $x_{c}=0.4$, where $\rho_{n}$ increases by almost an order of magnitude. This indicates that properties of SFS junctions depend not only on the electronic disorder (m.f.p.) but also on the magnetic disorder and, particularly, are affected by quantum fluctuations at the quantum phase transition reflected by the sign change of the AHE, Fig. 6(a).

From the comparison of $\rho_{x x 0}$ and $\rho_{n}$, Figs. 6(c) and 8(b), it is also seen that junction resistivity is several times larger than the film resistivity. This indicates that junction resistances are dominated by an additional resistance at $\mathrm{S} / \mathrm{F}$ interfaces due to a finite interface transparency $\beta<1$. For SNS junctions the interface transparency is reduced by a mismatch between Fermi velocities and Fermi surfaces (electronic band structures) of $\mathrm{S}$ and $\mathrm{N}$ metals $[58,59]$. For example, the transparency of $\mathrm{Nb} / \mathrm{Cu}$ interface was estimated to be $\beta \simeq 0.4$ [58]. The transparency of $\mathrm{S} / \mathrm{F}$ interfaces is further reduced due to spin imbalance, which affects the Andreev reflection of spinsinglet Cooper pairs from spin-polarized ferromagnet [1,4,5965]. The values $R_{n} A \sim 1 \times 10^{-10} \Omega \mathrm{cm}^{2}$ in our junctions, see Tables I-III, are comparable to the value $0.64 \times 10^{-10} \Omega \mathrm{cm}^{2}$ reported for $\mathrm{Nb} / \mathrm{Co}$ interfaces [65]. From Table III it can be seen that for $\mathrm{Nb} / \mathrm{Pt}_{1-x} \mathrm{Ni}_{x} / \mathrm{Nb}$ JJ's with $x \simeq 0.4$ and 0.6 , corresponding to quantum critical points of vanishing AHE, $\sigma_{\mathrm{AHE}}(T=0) \simeq 0$, see Fig. 6(a) and Ref. [44], the $R_{n} A$ value and thus the interface resistance greatly increases. Simultaneously the critical current density increases, leading to extraordinary large $I_{c} R_{n}$ products of several hundreds of $\mu \mathrm{V}$,
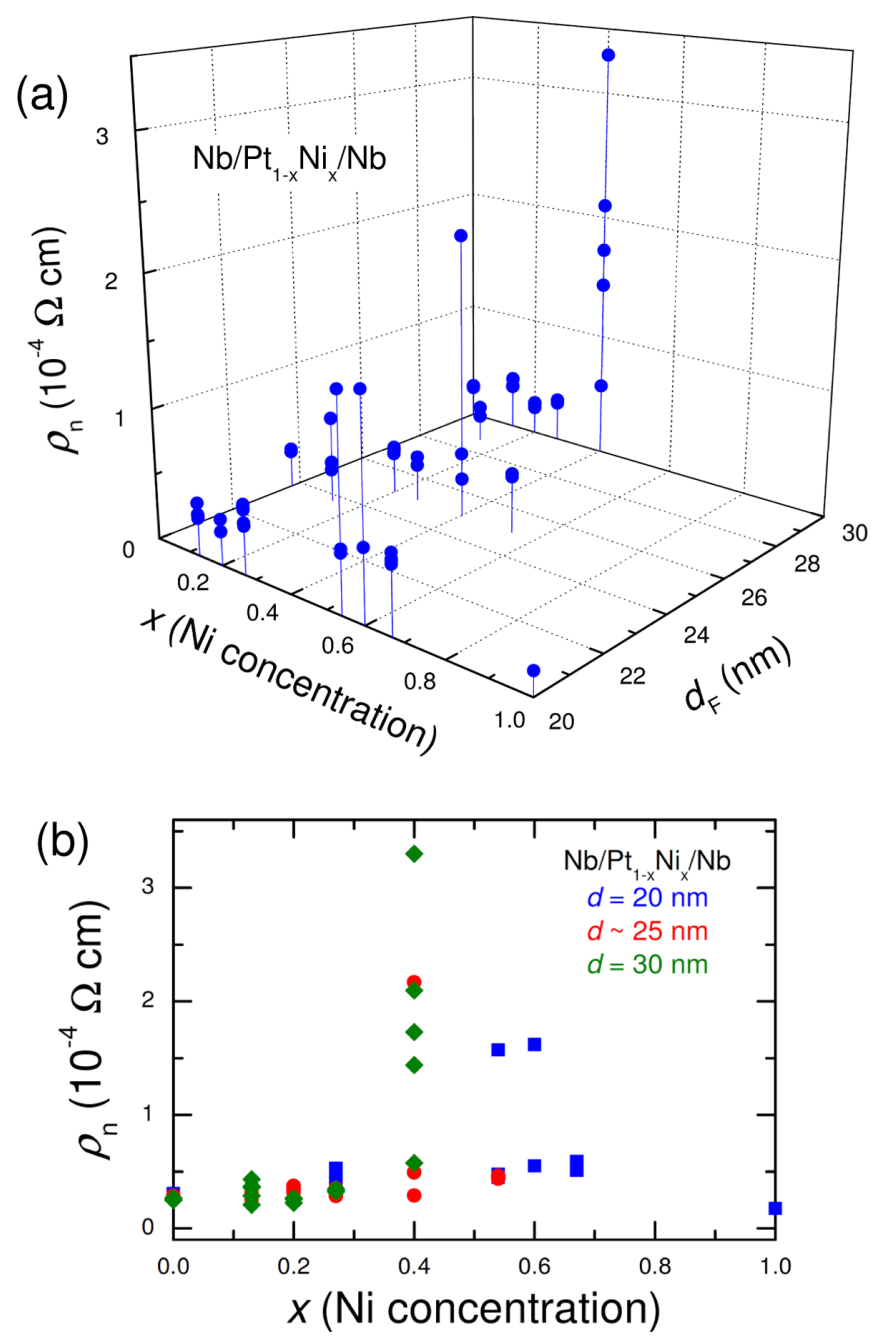

FIG. 8. (a) Three-dimensional plot of normal resistivities of $\mathrm{Nb} / \mathrm{Pt}_{1-x} \mathrm{Ni}_{x} / \mathrm{Nb}$ junctions versus $\mathrm{Ni}$ concentration and interlayer thickness. (b) Projection of the same data on the two-dimensional plot. Note sharp singularities at the critical concentration $x_{c}=0.4$ and a secondary maximum at $x \simeq 0.6$, corresponding to points of sign-reversal AHE in Fig. S3(a).

comparable to that for SINIS (I-insulator) junctions [66]. The origin of this phenomenon remains to be understood. So far we can only speculate that anomalous junction characteristics at these critical concentrations are related to quantum phase transitions occurring between ordered $L 1_{0}$ and $L 1_{2}$ phases with different magnetic properties [53,55,57,67]. For all our junctions, $R_{n}$ is dominated by $\mathrm{S} / \mathrm{F}$ interface resistances, consistent with earlier reports for other types of $\mathrm{S} / \mathrm{F}$ interfaces $[59,63,65]$. Therefore, there are significant barriers at $S / F$ interfaces, despite that the deposition of SFS trilayers occurred in one run without breaking vacuum.

\section{APPENDIX E: CLARIFICATION ABOUT EXTRACTION OF MAGNETIZATION CURVES FROM AJF ANALYSIS}

In the derivation of Eq. (1) we assumed that $M_{F}$ has an in-plane orientation. Due to the small thickness of F interlayers, they have negligibly small demagnetization factors. In this case the $\mathrm{F}$ layer does not generate magnetic fields at 
$\mathrm{S} / \mathrm{F}$ interfaces and, therefore, does not induce any additional flux in $\mathrm{S}$ electrodes. This leads to a simple separation of flux contributions from the $\mathrm{S}$ electrodes and $\mathrm{F}$ interlayer, represented by first and second terms in Eq. (1). Here $B$ in the first term does not contain $M_{F}$ (i.e., it is not $H+4 \pi M_{F}$ ) and differs from $H$ solely due to screening by superconducting currents and a finite demagnetization factor of S electrodes, just like in the nonmagnetic junction. Since for our junctions the total thickness of S layers $2 d_{N b}=400 \mathrm{~nm}$ is comparable to junction sizes, the demagnetization factor of electrodes is non-negligible and the difference between $B$ and $H$ can be sensible. Nevertheless, this does not affect the linearity of $\Phi(H)$ curves above the saturation field because $B \propto H$ in the presence of the demagnetization effect. Therefore, subtraction of the linear asymptotics, shown by dashed lines in the middle panels of Figs. 2(a)-2(c), remains unambiguous.

The distance between points in the AJF analysis is determined by the flux quantization field $\Delta H=\Phi_{0} / L \Lambda$. It is smaller for the hard axis orientation of the field, corresponding to the longest size of the junction $L$. Therefore, AJF curves for the hard axis, Figs. 2(c) and 2(d), are much more detailed than for the easy axis orientation, Figs. 2(a) and 2(b). Nevertheless, extraction of $M_{F}(H)$ for the hard axis orientation is complicated by two factors: First, magnetization reversal in the hard axis orientation occurs initially via coherent rotation of magnetization (without hysteresis), followed by a small flip, and continuing coherent rotation towards the saturated state [41,46,68,69]. Since the flip is smaller than $M_{\text {sat }}$, it does not allow direct extraction of $M_{\text {sat }}$ from the size of the magnetization jump. Second, since the length of the electrode $L \sim 1 \mu \mathrm{m}$ in the hard axis orientation is much larger than the London penetration depth of $S$ electrodes, $\lambda_{S} \simeq 100 \mathrm{~nm}$, junctions are prone to penetration of Abrikosov vortices, which greatly distort junction characteristics $[45,70]$. Therefore, the field range of our analysis is limited by the range of the Meissner state.

For the JJ with $d_{\mathrm{Ni}}=10 \mathrm{~nm}$, Fig. 2(c), the Meissner state persists up to the saturation state, and the straightforward subtraction of the high-field linear slope from the AJF curves, shown by the dashed line in the middle panel of Fig. 2(c), provides a magnetization loop with the expected saturation at high fields, as shown in the bottom panel of Fig. 2(c). It can be seen that saturation occurs at $H \simeq 1 \mathrm{kOe}$, which is consistent with that obtained using the first-order reversal curves analysis on the same junction, see Fig. 2(f) in Ref. [41].

For the JJ with $d_{\mathrm{Ni}}=20 \mathrm{~nm}$, Fig. 2(d), the field range is limited by the entrance of Abrikosov vortices. It is smaller than $1 \mathrm{kOe}$ and the saturation is presumably not reached, which does not allow unambiguous determination of the linear asymptotics. In this case we have chosen to assume $B=H$ in Eq. (1) and calculate the first linear term using the definition of the magnetic thickness, $\Lambda=d_{F}+\lambda_{S 1} \tanh \left(d_{S 1} / 2 \lambda_{S 1}\right)+$ $\lambda_{S 2} \tanh \left(d_{S 2} / 2 \lambda_{S 2}\right)$, where $d_{S 1,2}=200 \mathrm{~nm}$ are is the thicknesses and $\lambda_{S 1,2}=100 \mathrm{~nm}$ are the London penetration depths of the two $\mathrm{Nb}$ electrodes. The corresponding linear dependence $\Phi_{1}(H)$ is shown by the dashed line in the middle panel of Fig. 2(d). Thus obtained magnetization curve, $M_{\mathrm{Ni}}\left(H_{\perp}\right)$, shown in the bottom panel of Fig. 2(d), is in line with the expected magnetization curve for the hard axis orientation, as discussed above, and provides a correct value of $M_{\text {sat }}$.
[1] M. J. M. de Jong and C. W. J. Beenakker, Andreev Reflection in Ferromagnet-Superconductor Junctions, Phys. Rev. Lett. 74, 1657 (1995).

[2] E. A. Demler, G. B. Arnold, and M. R. Beasley, Superconducting proximity effects in magnetic metals, Phys. Rev. B 55, 15174 (1997).

[3] A. Kadigrobov, R. I. Shekhter, and M. Jonson, Quantum spin fluctuations as a source of long-range proximity effects in diffusive ferromagnet-superconductor structures, Europhys. Lett. 54, 394 (2001).

[4] B. P. Vodopyanov and L. R. Tagirov, Andreev conductance of a ferromagnet/superconductor point contact, JETP Lett. 77, 126 (2003).

[5] J. Kopu, M. Eschrig, J. C. Cuevas, and M. Fogelström, Transfer-matrix description of heterostructures involving superconductors and ferromagnets, Phys. Rev. B 69, 094501 (2004).

[6] A. I. Buzdin, Proximity effects in superconductor-ferromagnet heterostructures, Rev. Mod. Phys. 77, 935 (2005).

[7] F. S. Bergeret, A. F. Volkov, and K. B. Efetov, Odd triplet superconductivity and related phenomena in superconductorferromagnet structures, Rev. Mod. Phys. 77, 1321 (2005).

[8] M. Božović and Z. Radović, Ferromagnet-superconductor proximity effect: The clean limit, Europhys. Lett. 70, 513 (2005).
[9] Y. Asano, Y. Sawa, Y. Tanaka, and A. A. Golubov, Odd triplet superconductivity and related phenomena in superconductorferromagnet structures, Phys. Rev. B 76, 224525 (2007).

[10] M. A. Silaev, Possibility of a long-range proximity effect in a ferromagnetic nanoparticle, Phys. Rev. B 79, 184505 (2009).

[11] Ya. V. Fominov, A. A. Golubov, T. Yu. Karminskaya, M. Yu. Kupriyanov, R. G. Deminov, and L. R. Tagirov, Superconducting triplet spin valve, JETP Lett. 91, 308 (2010).

[12] F. Konschelle, J. Cayssol, and A. Buzdin, Long-range singlet proximity effect in ferromagnetic nanowires, Phys. Rev. B 82, 180509(R) (2010).

[13] M. Alidoust, J. Linder, G. Rashedi, T. Yokoyama, and A. Sudbø, Spin-polarized Josephson current in superconductor/ferromagnet/superconductor junctions with inhomogeneous magnetization, Phys. Rev. B 81, 014512 (2010).

[14] N. G. Pugach, M. Yu. Kupriyanov, E. Goldobin, R. Kleiner, and D. Koelle, Superconductor-insulator-ferromagnetsuperconductor Josephson junction: From the dirty to the clean limit, Phys. Rev. B 84, 144513 (2011).

[15] A. S. Mel'nikov, A. V. Samokhvalov, S. M. Kuznetsova, and A. I. Buzdin, Interference Phenomena and Long-Range Proximity Effect in Clean Superconductor-Ferromagnet Systems, Phys. Rev. Lett. 109, 237006 (2012). 
[16] S. H. Jacobsen and J. Linder, Giant triplet proximity effect in $\pi$-biased Josephson junctions with spin-orbit coupling, Phys. Rev. B 92, 024501 (2015).

[17] I. V. Bobkova, A. M. Bobkov, and M. A. Silaev, Gauge theory of the long-range proximity effect and spontaneous currents in superconducting heterostructures with strong ferromagnets, Phys. Rev. B 96, 094506 (2017).

[18] N. Klenov, Y. Khaydukov, S. Bakurskiy, R. Morari, I. Soloviev, V. Boian, T. Keller, M. Kupriyanov, A. Sidorenko, and B. Keimer, Periodic $\mathrm{Co} / \mathrm{Nb}$ pseudo spin valve for cryogenic memory, Beilstein J. Nanotechnol. 10, 833 (2019).

[19] V. T. Petrashov, I. A. Sosnin, I. Cox, A. Parsons, and C. Troadec, Giant Mutual Proximity Effects in Ferromagnetic/Superconducting Nanostructures, Phys. Rev. Lett. 83, 3281 (1999).

[20] V. V. Ryazanov, V. A. Oboznov, A. Yu. Rusanov, A. V. Veretennikov, A. A. Golubov, and J. Aarts, Coupling of Two Superconductors through a Ferromagnet: Evidence for a $\pi$ Junction, Phys. Rev. Lett. 86, 2427 (2001).

[21] J. Aumentado and V. Chandrasekhar, Mesoscopic ferromagnetsuperconductor junctions and the proximity effect, Phys. Rev. B 64, 054505 (2001).

[22] T. Kontos, M. Aprili, J. Lesueur, F. Genêt, B. Stephanidis, and R. Boursier, Josephson Junction through a Thin Ferromagnetic Layer: Negative Coupling, Phys. Rev. Lett. 89, 137007 (2002).

[23] V. A. Oboznov, V. V. Bol'ginov, A. K. Feofanov, V. V. Ryazanov, and A. I. Buzdin, Thickness Dependence of the Josephson Ground States of Superconductor-FerromagnetSuperconductor Junctions, Phys. Rev. Lett. 96, 197003 (2006).

[24] F. Born, M. Siegel, E. K. Hollmann, H. Braak, A. A. Golubov, D. Yu. Gusakova, and M. Yu. Kupriyanov, Multiple $0-\pi$ transitions in superconductor/insulator/ferromagnet/superconductor Josephson tunnel junctions, Phys. Rev. B 74, 140501(R) (2006).

[25] R. S. Keizer, S. T. B. Goennenwein, T. M. Klapwijk, G. Miao, G. Xiao, and A. Gupta, A spin triplet supercurrent through the half-metallic ferromagnet $\mathrm{CrO}_{2}$, Nature (London) 439, 825 (2006).

[26] J. W. A. Robinson, S. Piano, G. Burnell, C. Bell, and M. G. Blamire, Zero to $\pi$ transition in superconductor-ferromagnetsuperconductor junctions, Phys. Rev. B 76, 094522 (2007).

[27] A. A. Bannykh, J. Pfeiffer, V. S. Stolyarov, I. E. Batov, V. V. Ryazanov, and M. Weides, Josephson tunnel junctions with a strong ferromagnetic interlayer, Phys. Rev. B 79, 054501 (2009).

[28] J. W. A. Robinson, G. B. Halász, A. I. Buzdin, and M. G. Blamire, Enhanced Supercurrents in Josephson Junctions Containing Nonparallel Ferromagnetic Domains, Phys. Rev. Lett. 104, 207001 (2010).

[29] M. Flokstra, J. M. van der Knaap, and J. Aarts, Magnetic coupling in superconducting spin valves with strong ferromagnets, Phys. Rev. B 82, 184523 (2010).

[30] J. Wang, M. Singh, M. Tian, N. Kumar, B. Liu, C. Shi, J. K. Jain, N. Samarth, T. E. Mallouk, and M. H. W. Chan, Interplay between superconductivity and ferromagnetism in crystalline nanowires, Nat. Phys. 6, 389 (2010).
[31] K. M. Boden, W. P. Pratt, Jr., and N. O. Birge, Proximity-induced density-of-states oscillations in a superconductor/strong-ferromagnet system, Phys. Rev. B 84, 020510(R) (2011).

[32] V. V. Bol'ginov, V. S. Stolyarov, D. S. Sobanin, A. L. Karpovich, and V. V. Ryazanov, Magnetic switches based on $\mathrm{NbPdFeNb}$ Josephson junctions with a magnetically soft ferromagnetic interlayer, JETP Lett. 95, 366 (2012).

[33] T. Golod, A. Rydh, V. M. Krasnov, I. Marozau, M. A. UribeLaverde, D. K. Satapathy, Th. Wagner, and C. Bernhard, High bias anomaly in $\mathrm{YBa}_{2} \mathrm{Cu}_{3} \mathrm{O}_{7-x} / \mathrm{LaMnO}_{3+\delta} / \mathrm{YBa}_{2} \mathrm{Cu}_{3} \mathrm{O}_{7-x}$ superconductor/ferromagnetic insulator/superconductor junctions: Evidence for a long-range superconducting proximity effect through the conduction band of a ferromagnetic insulator, Phys. Rev. B 87, 134520 (2013).

[34] B. Baek, W. H. Rippard, S. P. Benz, and S. E. Russek, and P. D. Dresselhaus, Hybrid superconducting-magnetic memory device using competing order parameters, Nat. Commun. 5, 3888 (2014).

[35] M. Kompaniiets, O. V. Dobrovolskiy, C. Neetzel, E. Begun, F. Porrati, W. Ensinger, and M. Huth, Proximity-induced superconductivity in crystalline $\mathrm{Cu}$ and $\mathrm{Co}$ nanowires and nanogranular Co structures, J. Appl. Phys. 116, 073906 (2014).

[36] A. Iovan, T. Golod, and V. M. Krasnov, Controllable generation of a spin-triplet supercurrent in a Josephson spin valve, Phys. Rev. B 90, 134514 (2014).

[37] D. Lenk, V. I. Zdravkov, J.-M. Kehrle, G. Obermeier, A. Ullrich, R. Morari, H.-A. Krug von Nidda, C. Müller, M. Yu. Kupriyanov, A. S. Sidorenko, S. Horn, R. G. Deminov, L. R. Tagirov, and R. Tidecks, Thickness dependence of the triplet spin-valve effect in superconductor-ferromagnetferromagnet heterostructures, Beilstein J. Nanotechnol. 7, 957 (2016).

[38] K. Lahabi, M. Amundsen, J. A. Ouassou, E. Beukers, M. Pleijster, J. Linder, and P. Alkemade, and J. Aarts, Controlling supercurrents and their spatial distribution in ferromagnets, Nat. Commun. 8, 2056 (2017).

[39] N. O. Birge, Spin-triplet supercurrents in Josephson junctions containing strong ferromagnetic materials, Phil. Trans. R. Soc. A 376, 20150150 (2018).

[40] O. V. Skryabina, S. N. Kozlov, S. V. Egorov, A. A. Klimenko, V. V. Ryazanov, S. V. Bakurskiy, M. Yu. Kupriyanov, N. V. Klenov, I. I. Soloviev, A. A. Golubov, K. S. Napolskii, I. A. Golovchanskiy, D. Roditchev, and V. S. Stolyarov, Anomalous magneto-resistance of Ni-nanowire/Nb hybrid system, Sci. Rep. 9, 14470 (2019).

[41] O. M. Kapran, A. Iovan, T. Golod, and V. M. Krasnov, Observation of the dominant spin-triplet supercurrent in Josephson spin valves with strong Ni ferromagnets, Phys. Rev. Research 2, 013167 (2020).

[42] J. Linder and A. V. Balatsky, Odd-frequency superconductivity, Rev. Mod. Phys. 91, 045005 (2019).

[43] T. Golod, A. Rydh, and V. M. Krasnov, Anomalous Hall effect in NiPt thin films, J. Appl. Phys. 110, 033909 (2011).

[44] T. Golod, A. Rydh, P. Svedlindh, and V. M. Krasnov, Antiordinary Hall effect near the ferromagnetic quantum phase transition in $\mathrm{Ni}_{x} \mathrm{Pt}_{1-x}$ thin films, Phys. Rev. B 87, 104407 (2013). 
[45] T. Golod, A. Rydh, and V. M. Krasnov, Detection of the Phase Shift from a Single Abrikosov Vortex, Phys. Rev. Lett. 104, 227003 (2010).

[46] A. Iovan and V. M. Krasnov, Signatures of the spin-triplet current in a Josephson spin valve: A micromagnetic analysis, Phys. Rev. B 96, 014511 (2017).

[47] V. M. Krasnov, V. A. Oboznov, and N. F. Pedersen, Fluxon dynamics in long Josephson junctions in the presence of a temperature gradient or spatial nonuniformity, Phys. Rev. B 55, 14486 (1997).

[48] A. T. Aldred, Temperature dependence of the magnetization of nickel, Phys. Rev. B 11, 2597 (1975).

[49] H. Danan, A. Herr, and A. J. P. Meyer, New determinations of the saturation magnetization of nickel and iron, J. Appl. Phys. 39, 669 (1968).

[50] A. A. Golubov, M. Yu. Kupriyanov, and E. Il'ichev, The current-phase relation in Josephson junctions, Rev. Mod. Phys. 76, 411 (2004).

[51] A. A. Golubov and V. M. Krasnov, The first critical field, $H_{c 1}^{\perp}$, and the penetration depth in dirty superconducting $\mathrm{S} / \mathrm{N}$ multilayers, Physica C 196, 177 (1992).

[52] For in-plane transport the electronic m.f.p. is limited by the film thickness due to interface scattering. However, here we consider perpendicular transport, for which such scattering is irrelevant. Therefore, the effective m.f.p. in the perpendicular direction is not limited by the film thickness.

[53] C. E. Dahmani, M. C. Cadeville, J. M. Sanchez, and J. L. Morán-López, Ni-Pt Phase Diagram: Experiment and Theory, Phys. Rev. Lett. 55, 1208 (1985).

[54] M. J. Besnus and A. Herr, Transition from ferromagnetism to paramagnetism in Ni-Pt alloys, Phys. Lett. A 39, 83 (1972).

[55] A. Hizi, H. Garbouj, C. Mottet, and M. Said, Chemical ordering and surface segregation in $\mathrm{Ni}_{1-c} \mathrm{Pt}_{c}$ system: A theoretical study from the alloys to the nanoalloys, Results Phys. 14, 102493 (2019).

[56] N. Nagaosa, J. Sinova, S. Onoda, A. H. MacDonald, and N. P. Ong, Anomalous Hall effect, Rev. Mod. Phys. 82, 1539 (2010).

[57] H. Zhang, S. Blügel, and Yu. Mokrousov, Anisotropic intrinsic anomalous Hall effect in ordered 3dPt alloys, Phys. Rev. B 84, 024401 (2011).

[58] V. M. Krasnov, V. A. Oboznov, and V. V. Ryazanov, Anomalous temperature dependence of $H_{c 1}^{\perp}$ in superconducting $\mathrm{Nb} / \mathrm{Cu}$ multilayer, Physica C 196, 335 (1992).
[59] S. K. Upadhyay, A. Palanisami, R. N. Louie, and R. A. Buhrman, Probing Ferromagnets with Andreev Reflection, Phys. Rev. Lett. 81, 3247 (1998).

[60] J. Aarts, J. M. E. Geers, E. Brück, A. A. Golubov, and R. Coehoorn, Interface transparency of superconductor/ferromagnetic multilayers, Phys. Rev. B 56, 2779 (1997).

[61] V. I. Fal'ko, C. J. Lambert, and A. F. Volkov, Andreev reflections and magnetoresistance in ferromagnet/superconductor mesoscopic structures, JETP Lett. 69, 532 (1999).

[62] A. A. Golubov, Interface resistance in ferromagnetrsuperconductor junctions, Physica C 326-327, 46 (1999).

[63] O. Bourgeois, P. Gandit, A. Sulpice, J. Lesueur, and X. Grison, Transport in superconductor/ferromagnet/superconductor junctions dominated by interface resistance, Phys. Rev. B 63, 064517 (2001).

[64] K. Xia, P. J. Kelly, G. E. W. Bauer, and I. Turek, SpinDependent Transparency of Ferromagnet/Superconductor Interfaces, Phys. Rev. Lett. 89, 166603 (2002).

[65] S. F. Lee, S. Y. Huang, J. H. Kuo, Y. A. Lin, L. K. Lin, and Y. D. Yao, Quantitative analysis of interface resistance in $\mathrm{Co} / \mathrm{Nb}$ multilayers for normal and superconducting Nb, J. Appl. Phys. 93, 8212 (2003).

[66] A. A. Golubov, E. P. Houwman, J. G. Gijsbertsen, V. M. Krasnov, J. Flokstra, and H. Rogalla, Proximity effect in superconductor-insulator-superconductor Josephson tunnel junctions: Theory and experiment, Phys. Rev. B 51, 1073 (1995).

[67] M. Chen, Z. Shi, W. J. Xu, X. X. Zhang, J. Du, and S. M. Zhou, Tuning anomalous hall conductivity in ${ }_{0} \mathrm{FePt}$ films by long range chemical ordering, Appl. Phys. Lett. 98, 082503 (2011).

[68] Y. Henry, A. Iovan, J.-M. George, and L. Piraux, Statistical analysis of the magnetization processes in arrays of electrodeposited ferromagnetic nanowires, Phys. Rev. B 66, 184430 (2002).

[69] Yu. P. Ivanov, O. Iglesias-Freire, E. V. Pustovalov, O. Chubykalo-Fesenko, and A. Asenjo, Magnetic configurations of $\mathrm{Co}(111)$ nanostripes with competing shape and crystalline anisotropies, Phys. Rev. B 87, 184410 (2013).

[70] T. Golod, A. Pagliero, and V. M. Krasnov, Two mechanisms of Josephson phase shift generation by an Abrikosov vortex, Phys. Rev. B 100, 174511 (2019). 